J. Korean Math. Soc. 51 (2014), No. 6, pp. 1269-1289

http://dx.doi.org/10.4134/JKMS.2014.51.6.1269

\title{
ON SOME THETA CONSTANTS AND CLASS FIELDS
}

\author{
Dong Hwa SHin
}

\begin{abstract}
We first find a sufficient condition for a product of theta constants to be a Siegel modular function of a given even level. And, when $K_{(2 p)}$ denotes the ray class field of $K=\mathbb{Q}\left(e^{2 \pi i / 5}\right)$ modulo $2 p$ for an odd prime $p$, we describe a subfield of $K_{(2 p)}$ generated by the special value of a certain theta constant by using Shimura's reciprocity law.
\end{abstract}

\section{Introduction}

Let $N(\geq 2)$ be an integer and $\mathfrak{F}_{N}$ be the field of meromorphic modular functions of level $N$ whose Fourier coefficients lie in the $N$ th cyclotomic field $([9]$ or $[7, \S 6.3])$. For a vector $\left[\begin{array}{l}r \\ s\end{array}\right] \in(1 / N) \mathbb{Z}^{2}-\mathbb{Z}^{2}$ the Siegel function $g_{\left[\begin{array}{l}r \\ s\end{array}\right]}(\tau)$ is defined on the complex upper half-plane $\mathbb{H}$ by the following infinite product

$$
\begin{aligned}
g_{\left[\begin{array}{l}
r \\
s
\end{array}\right]}(\tau)=- & q^{(1 / 2)\left(r^{2}-r+1 / 6\right)} e^{\pi i s(r-1)}\left(1-q^{r} e^{2 \pi i s}\right) \\
& \times \prod_{n=1}^{\infty}\left(1-q^{n+r} e^{2 \pi i s}\right)\left(1-q^{n-r} e^{-2 \pi i s}\right)(\tau \in \mathbb{H}),
\end{aligned}
$$

where $q=e^{2 \pi i \tau}$ and $i=\sqrt{-1}$. Let $\{m(r, s)\}_{\left[\begin{array}{l}r \\ s\end{array}\right] \in(1 / N) \mathbb{Z}^{2}-\mathbb{Z}^{2}}$ be a family of integers such that $m(r, s) \neq 0$ only for finitely many vectors $\left[\begin{array}{l}r \\ s\end{array}\right]$. Kubert and Lang [6, Chapter 3, Theorem 5.3] showed that if $\{m(r, s)\}_{\left[\begin{array}{l}r \\ s\end{array}\right]}$ satisfies the quadratic relation modulo $N$, namely

$$
\begin{aligned}
& \sum_{\left[\begin{array}{l}
r \\
s
\end{array}\right]} m(r, s)(N r)^{2} \equiv \sum_{\left[\begin{array}{l}
r \\
s
\end{array}\right]} m(r, s)(N s)^{2} \equiv 0 \quad(\bmod \operatorname{gcd}(2, N) \cdot N), \\
& \sum_{\left[\begin{array}{l}
r \\
s
\end{array}\right]} m(r, s)(N r)(N s) \equiv 0 \quad(\bmod N),
\end{aligned}
$$

Received March 13, 2014.

2010 Mathematics Subject Classification. Primary 11F46; Secondary 11G15, 14 K25.

Key words and phrases. CM-fields, Shimura's reciprocity law, theta functions.

This work was supported by the NRF of Korea grant funded by the MEST (20142064001). 
and 12 divides $\operatorname{gcd}(12, N) \cdot \sum_{\left[\begin{array}{l}r \\ s\end{array}\right]} m(r, s)$, then the product $\zeta \prod_{\left[\begin{array}{l}r \\ s\end{array}\right]} g_{\left[\begin{array}{l}r \\ s\end{array}\right]}(\tau)^{m(r, s)}$ belongs to $\mathfrak{F}_{N}$, where $\zeta=\prod_{\left[\begin{array}{l}r \\ s\end{array}\right]} e^{\pi i s(1-r) m(r, s)}$. In particular, $g_{\left[\begin{array}{l}r \\ s\end{array}\right]}(\tau)^{12 N}$ belongs to $\mathfrak{F}_{N}$ for any vector $\left[\begin{array}{l}r \\ s\end{array}\right] \in(1 / N) \mathbb{Z}^{2}-\mathbb{Z}^{2}$, which depends only on $\left[\begin{array}{l}r \\ s\end{array}\right]$ $\left(\bmod \mathbb{Z}^{2}\right)$. And, the group $\mathrm{GL}_{2}(\mathbb{Z} / N \mathbb{Z})$ acts on the family

by the rule

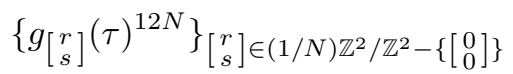

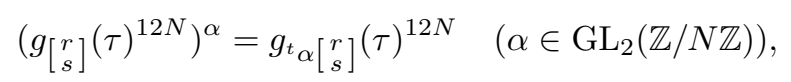

where ${ }^{t} \alpha$ stands for the transpose of $\alpha$ [6, Chapter 2, Proposition 1.3].

Now, let $K$ be an imaginary quadratic field and $\mathcal{O}_{K}=\mathbb{Z}[\theta]$ be its ring of integers with $\theta \in \mathbb{H}$. For a positive integer $N$ we denote the ray class field of $K$ modulo $N$ by $K_{(N)}$. The main theorem of complex multiplication ensures that $K_{(N)}$ is generated by the singular values $f(\theta)$ for all $f \in \mathfrak{F}_{N}$ which are finite at $\theta[7$, Chapter 10, Corollary to Theorem 1]. And, by using Shimura's reciprocity law $[9$, Theorem 6.31] Jung et al. recently showed in [3] that if $K \neq \mathbb{Q}(\sqrt{-1}), \mathbb{Q}(\sqrt{-3})$ and $N \geq 2$, then $K_{(N)}$ is generated by the singular value $g_{\left[\begin{array}{c}0 \\ 1 / N\end{array}\right]}(\theta)^{12 N}$ over $K$.

In this paper we shall attempt to find higher dimensional analogues of these results. Siegel modular functions of level $N(\geq 1)$ defined on the Siegel upper half-space $\mathbb{H}_{g}(g \geq 2)$ are certain multi-variable functions which generalize meromorphic modular functions of one variable $(\S 2)$. As in the case of modular functions, the action of the general symplectic group $\operatorname{GSp}_{2 g}(\mathbb{Z} / N \mathbb{Z})$ on the Siegel modular functions of level $N$ was investigated by Shimura (Proposition 2.1). If $\mathbf{r}, \mathbf{s} \in(1 / N) \mathbb{Z}^{g}$, then the theta constant

$$
\Phi_{\left[\begin{array}{l}
\mathbf{r} \\
\mathbf{s}
\end{array}\right]}(Z)=\frac{\sum_{\mathbf{x} \in \mathbb{Z}^{g}} e\left({ }^{t}(\mathbf{x}+\mathbf{r}) Z(\mathbf{x}+\mathbf{r}) / 2+{ }^{t}(\mathbf{x}+\mathbf{r}) \mathbf{s}\right)}{\sum_{\mathbf{x} \in \mathbb{Z}^{g}} e\left({ }^{t} \mathbf{x} Z \mathbf{x} / 2\right)}
$$

is a typical example of Siegel modular functions (of level $2 N^{2}$ ) (§4). We shall first give a sufficient condition for a product of theta constants to be a Siegel modular function of level $N$ when $N$ is even (Theorem 4.3). And, we shall further show that a certain subgroup of $\mathrm{GSp}_{2 g}(\mathbb{Z} / N \mathbb{Z})$ acts on the family $\left\{\Phi_{\left[\begin{array}{l}\mathbf{r} \\ \mathbf{s}\end{array}\right]}(Z)^{2 N^{2}}\right\}_{\mathbf{r}, \mathbf{s} \in(1 / N) \mathbb{Z}^{g} / \mathbb{Z}^{g}}$ in a natural way, namely

$$
\left(\Phi_{\left[\begin{array}{l}
\mathbf{r} \\
\mathbf{s}
\end{array}\right]}(Z)^{2 N^{2}}\right)^{\alpha}=\Phi_{t_{\alpha}\left[\begin{array}{r}
\mathbf{r} \\
\mathbf{s}
\end{array}\right]}(Z)^{2 N^{2}}
$$

(Theorem 5.2).

On the other hand, let $K$ be a CM-field, $K^{*}$ be its reflex field and $Z_{0}$ be the associated CM-point $(\S 6)$. The theory of complex multiplication for polarized abelian varieties of higher dimension developed by Shimura claims that if $f$ is a Siegel modular function that is finite at $Z_{0}$, then the special value $f\left(Z_{0}\right)$ lies in some abelian extension of $K^{*}$. Furthermore, Shimura's reciprocity law describes Galois actions on $f\left(Z_{0}\right)$ in terms of actions of general symplectic 
groups on $f$ (Proposition 6.2). Here, we focus on the case where $K=\mathbb{Q}\left(e^{2 \pi i / 5}\right)$. For an odd prime $p$ let $K_{(2 p)}$ and $K_{\left(2 p^{2}\right)}$ be the ray class fields of $K$ modulo $2 p$ and $2 p^{2}$, respectively. Komatsu considered in [5] a certain intermediate field $L$ of $K_{\left(2 p^{2}\right)} / K_{(2 p)}$ with $\left[L: K_{(2 p)}\right]=p^{3}$ and provided a normal basis of $L$ over $K_{(2 p)}$. Unlike Komatsu's work, however, we shall examine the field $K\left(\Phi\left[\begin{array}{c}1 / p \\ 0 \\ 0 \\ 0\end{array}\right]\left(Z_{0}\right)^{2 p^{2}}\right)$ as a subfield of $K_{(2 p)}$ (Theorems 7.3 and 7.4 ). To this end we shall utilize transformation formulas of theta functions (Propositions 3.1 and 3.2) together with Shimura's reciprocity law.

And, we shall also present some ideas of combining two generators of an abelian extension to get a primitive generator (Theorems 8.2 and 8.4).

\section{Siegel modular forms}

We shall introduce necessary facts about Siegel modular forms, and explain actions of general symplectic groups on the Siegel modular functions whose Fourier coefficients lie in some cyclotomic fields.

Let $g(\geq 2)$ be a positive integer and

$$
J=\left[\begin{array}{cc}
0 & -I_{g} \\
I_{g} & 0
\end{array}\right] .
$$

Given a commutative ring $R$ with unity we let

$$
\operatorname{GSp}_{2 g}(R)=\left\{\alpha \in \operatorname{Mat}_{2 g}(R) \mid{ }^{t} \alpha J \alpha=\nu J \text { for some } \nu \in R^{\times}\right\} .
$$

Considering $\nu$ as a homomorphism $\operatorname{GSp}_{2 g}(R) \rightarrow R^{\times}$we denote its kernel by $\mathrm{Sp}_{2 g}(R)$, namely

$$
\operatorname{Sp}_{2 g}(R)=\left\{\alpha \in \operatorname{Mat}_{2 g}(R) \mid{ }^{t} \alpha J \alpha=J\right\} .
$$

We further define a homomorphism $\iota: R^{\times} \rightarrow \operatorname{GSp}_{2 g}(R)$ by

$$
\iota(a)=\left[\begin{array}{cc}
I_{g} & 0 \\
0 & a^{-1} I_{g}
\end{array}\right] .
$$

One can then readily show that $\nu(\iota(a))=a^{-1}$.

The Siegel upper half-space $\mathbb{H}_{g}$ is defined by

$$
\mathbb{H}_{g}=\left\{Z \in \operatorname{Mat}_{g}(\mathbb{C}) \mid{ }^{t} Z=Z, \operatorname{Im}(Z) \text { is positive definite }\right\} .
$$

Then, it is well-known that $\operatorname{Sp}_{2 g}(\mathbb{Z})$ acts on $\mathbb{H}_{g}$ by

$$
\left[\begin{array}{ll}
A & B \\
C & D
\end{array}\right](Z)=(A Z+B)(C Z+D)^{-1}
$$

where $A, B, C, D$ are $g \times g$ block matrices [4, $\S 1$, Proposition 1]. Let $N(\geq 1)$ and $k$ be integers, and define the group

$$
\Gamma(N)=\left\{\gamma \in \operatorname{Sp}_{2 g}(\mathbb{Z}) \mid \gamma \equiv I_{2 g} \quad(\bmod N)\right\}
$$


A holomorphic function $f: \mathbb{H}_{g} \rightarrow \mathbb{C}$ is called a Siegel modular form of weight $k$ and level $N$, if

$$
f(\gamma(Z))=\operatorname{det}(C Z+D)^{k} f(Z) \quad \text { for every } \gamma=\left[\begin{array}{ll}
A & B \\
C & D
\end{array}\right] \in \Gamma(N) .
$$

For $z \in \mathbb{C}$, we set

$$
e(z)=e^{2 \pi i z} .
$$

As a consequence of Köecher's principle, a Siegel modular form $f$ can be written as

$$
f(Z)=\sum_{\xi} c(\xi) e(\operatorname{tr}(\xi Z) / N) \quad(c(\xi) \in \mathbb{C}),
$$

where $\xi$ runs over all $g \times g$ positive semi-definite symmetric matrices over half integers with integral diagonal entries [4, $\S 4$, Theorem 1]. This expansion is called the Fourier expansion of $f$ with Fourier coefficients $c(\xi)$. Note that if $\xi=\left[\xi_{j k}\right]_{1 \leq j, k \leq g}$ and $Z=\left[Z_{j k}\right]_{1 \leq j, k \leq g}$, then

$$
\operatorname{tr}(\xi Z)=\sum_{j=1}^{g} \xi_{j j} Z_{j j}+2 \sum_{1 \leq j<k \leq g} \xi_{j k} Z_{j k}
$$

from which it follows that

$$
f(Z)=\sum_{\xi} c(\xi)\left(\prod_{j=1}^{g} e\left(\xi_{j j} Z_{j j} / N\right) \prod_{1 \leq j<k \leq g} e\left(2 \xi_{j k} Z_{j k} / N\right)\right) .
$$

Letting $\zeta_{N}=e(1 / N)$ we consider the field

$$
\mathcal{F}_{N}=\left\{\begin{array}{l|l}
g_{1} / g_{2} & \begin{array}{l}
g_{1} \text { and } g_{2}(\neq 0) \text { are Siegel modular forms of the same weight such that } \\
g_{1} / g_{2} \text { is invariant under } \Gamma(N) \text { and its Fourier coefficients lie in } \mathbb{Q}\left(\zeta_{N}\right)
\end{array}
\end{array}\right\} .
$$

Proposition 2.1. (i) $\iota\left((\mathbb{Z} / N \mathbb{Z})^{\times}\right)$acts on $\mathcal{F}_{N}$ as follows: If $a \in(\mathbb{Z} / N \mathbb{Z})^{\times}$and $f=\sum_{\xi} c(\xi) e(\operatorname{tr}(\xi Z) / N) \in \mathcal{F}_{N}$, then

$$
f^{\iota(a)}=\sum_{\xi} c(\xi)^{\rho(a)^{-1}} e(\operatorname{tr}(\xi Z) / N),
$$

where $\rho(a)$ is an endomorphism of $\mathbb{Q}\left(\zeta_{N}\right)$ induced from the map $\zeta_{N} \mapsto \zeta_{N}^{a}$. then

(ii) $\mathrm{Sp}_{2 g}(\mathbb{Z})$ acts on $\mathcal{F}_{N}$ by compositions, that is, if $\gamma \in \mathrm{Sp}_{2 g}(\mathbb{Z})$ and $f \in \mathcal{F}_{N}$,

$$
f^{\gamma}=f \circ \gamma .
$$

(iii) $\mathrm{GSp}_{2 g}(\mathbb{Z} / N \mathbb{Z})$ acts on $\mathcal{F}_{N}$ as follows: Let $\alpha \in \mathrm{GSp}_{2 g}(\mathbb{Z} / N \mathbb{Z})$ and $f \in$ $\mathcal{F}_{N}$. Set $a=\nu(\alpha) \in(\mathbb{Z} / N \mathbb{Z})^{\times}$and $\gamma=\iota(a) \alpha \in \operatorname{Sp}_{2 g}(\mathbb{Z} / N \mathbb{Z})$. Lift $\gamma$ to $\gamma_{0} \in \mathrm{Sp}_{2 g}(\mathbb{Z})$. Then,

$$
f^{\alpha}=\left(f^{\iota(a)^{-1}}\right)^{\gamma_{0}} .
$$

Proof. See $[11, \S 1]$. 


\section{Theta functions}

We shall briefly review fundamental transformation formulas of theta functions.

Let $g(\geq 2)$ be an integer, $\mathbf{u} \in \mathbb{C}^{g}, Z \in \mathbb{H}_{g}$ and $\mathbf{r}, \mathbf{s} \in \mathbb{R}^{g}$. We define a (classical) theta function by

$$
\Theta(\mathbf{u}, Z ; \mathbf{r}, \mathbf{s})=\sum_{\mathbf{x} \in \mathbb{Z}^{g}} e\left({ }^{t}(\mathbf{x}+\mathbf{r}) Z(\mathbf{x}+\mathbf{r}) / 2+{ }^{t}(\mathbf{x}+\mathbf{r})(\mathbf{u}+\mathbf{s})\right)
$$

which is a holomorphic function on $Z$. Since $\mathbf{x}$ can be replaced by $-\mathbf{x}$ in the above summation, we get the relation

$$
\Theta(-\mathbf{u}, Z ;-\mathbf{r},-\mathbf{s})=\Theta(\mathbf{u}, Z ; \mathbf{r}, \mathbf{s}) .
$$

Proposition 3.1. If $\mathbf{a}, \mathbf{b} \in \mathbb{Z}^{g}$, then we have the translation formula

$$
\Theta(\mathbf{u}, Z ; \mathbf{r}+\mathbf{a}, \mathbf{s}+\mathbf{b})=e\left({ }^{t} \mathbf{r b}\right) \Theta(\mathbf{u}, Z ; \mathbf{r}, \mathbf{s}) .
$$

Proof. See [10, p. $676(13)]$.

For a square matrix $\alpha$ we denote by $\{\alpha\}$ the column vector whose components are the diagonal elements of $\alpha$.

Proposition 3.2. Let $\gamma=\left[\begin{array}{cc}A & B \\ C & D\end{array}\right] \in \operatorname{Sp}_{2 g}(\mathbb{Z})$ such that $\left\{{ }^{t} A C\right\},\left\{{ }^{t} B D\right\} \in 2 \mathbb{Z}^{g}$. We get the transformation formula

$$
\begin{aligned}
& \Theta\left({ }^{t}(C Z+D)^{-1} \mathbf{u}, \gamma(Z) ; \mathbf{r}, \mathbf{s}\right) \\
= & \lambda_{\gamma} e\left(\left({ }^{t} \mathbf{r s}-{ }^{t} \mathbf{r}^{\prime} \mathbf{s}^{\prime}\right) / 2\right) \operatorname{det}(C Z+D)^{1 / 2} e\left(\left({ }^{t} \mathbf{u}(C Z+D)^{-1} C \mathbf{u}\right) / 2\right) \Theta\left(\mathbf{u}, Z ; \mathbf{r}^{\prime}, \mathbf{s}^{\prime}\right),
\end{aligned}
$$
where $\lambda_{\gamma}$ is a constant of absolute value 1 depending on $\gamma$ and the choice of the branch of $\operatorname{det}(C Z+D)^{1 / 2}$, and $\left[\begin{array}{l}\mathbf{r}^{\prime} \\ \mathbf{s}^{\prime}\end{array}\right]={ }^{t} \gamma\left[\begin{array}{l}\mathbf{r} \\ \mathbf{s}\end{array}\right]$.

Proof. See [10, Proposition 1.3].

And, let

$$
\Sigma_{-}=\left\{\left[\begin{array}{l}
\mathbf{r} \\
\mathbf{s}
\end{array}\right] \in \mathbb{Q}^{2 g} \mid \mathbf{r}, \mathbf{s} \in(1 / 2) \mathbb{Z}^{g} \text { and } e\left(2^{t} \mathbf{r s}\right)=-1\right\} .
$$

Proposition 3.3. Let $\mathbf{r}, \mathbf{s} \in \mathbb{Q}^{g}$. Then, $\Theta(\mathbf{0}, Z ; \mathbf{r}, \mathbf{s})$ represents the zero function on $Z$ if and only if $\left[\begin{array}{l}\mathbf{r} \\ \mathbf{s}\end{array}\right] \in \Sigma_{-}$.

Proof. See [2, Theorem 2].

\section{Modularity of theta constants}

In this section we shall find a sufficient condition for a product of theta constants to be a Siegel modular function of a given even level.

Let $N$ and $g(\geq 2)$ be positive integers and $\mathbf{r}, \mathbf{s} \in(1 / N) \mathbb{Z}^{g}$. We define a theta constant by

$$
\Phi_{\left[\begin{array}{l}
\mathbf{r} \\
\mathbf{s}
\end{array}\right]}(Z)=\frac{\Theta(\mathbf{0}, Z ; \mathbf{r}, \mathbf{s})}{\Theta(\mathbf{0}, Z ; \mathbf{0}, \mathbf{0})} \quad\left(Z \in \mathbb{H}_{g}\right)
$$


which is a nonzero function whenever we assume $\left[\begin{array}{l}\mathbf{r} \\ \mathbf{s}\end{array}\right] \notin \Sigma_{-}$by Proposition 3.3. It belongs to $\mathcal{F}_{2 N^{2}}$ by Propositions 3.1, 3.2 and the definition (1) (or [10, Proposition 7$]$ ). We get by (2) that

$$
\Phi_{\left[\begin{array}{c}
-\mathbf{r} \\
-\mathbf{s}
\end{array}\right]}(Z)=\Phi_{\left[\begin{array}{l}
\mathbf{r} \\
\mathbf{s}
\end{array}\right]}(Z) .
$$

Lemma 4.1. Let $a \in\left(\mathbb{Z} / 2 N^{2} \mathbb{Z}\right)^{\times}$. The action of

$$
\iota\left(a^{-1}\right)=\left[\begin{array}{cc}
I_{g} & 0 \\
0 & a I_{g}
\end{array}\right] \in \mathrm{GSp}_{2 g}\left(\mathbb{Z} / 2 N^{2} \mathbb{Z}\right)
$$

on $\Phi_{\left[\begin{array}{l}\mathbf{r} \\ \mathbf{s}\end{array}\right]}(Z)$ can be described as

$$
\Phi_{\left[\begin{array}{l}
\mathbf{r} \\
\mathbf{s}
\end{array}\right]}(Z)^{\iota\left(a^{-1}\right)}=\Phi_{\left[\begin{array}{c}
\mathbf{r} \\
a \mathbf{s}
\end{array}\right]}(Z)=\Phi_{t_{\iota\left(a^{-1}\right)}\left[\begin{array}{r}
\mathbf{r} \\
\mathbf{s}
\end{array}\right]}(Z) .
$$

Proof. We see from Proposition 3.1 that $\Phi_{\left[\begin{array}{c}\mathbf{r} \\ a \mathbf{s}\end{array}\right]}(Z)$ and $\Phi_{t_{\iota\left(a^{-1}\right)}\left[\begin{array}{l}\mathbf{r} \\ \mathbf{s}\end{array}\right]}(Z)$ are welldefined. And, it follows from the definition (1) that

$$
\begin{aligned}
& \Phi_{\left[\begin{array}{l}
\mathbf{r} \\
\mathbf{s}
\end{array}\right]}(Z)^{\iota\left(a^{-1}\right)} \\
= & \left(\frac{\sum_{\mathbf{x} \in \mathbb{Z}^{g}} e\left({ }^{t}(\mathbf{x}+\mathbf{r}) \mathbf{s}\right) e\left({ }^{t}(\mathbf{x}+\mathbf{r}) Z(\mathbf{x}+\mathbf{r}) / 2\right)}{\sum_{\mathbf{x} \in \mathbb{Z}^{g}} e\left(\left({ }^{(} \mathbf{x} Z \mathbf{x}\right) / 2\right)}\right)^{\iota\left(a^{-1}\right)} \\
= & \frac{\sum_{\mathbf{x} \in \mathbb{Z}^{g}} e\left({ }^{t}(\mathbf{x}+\mathbf{r}) a \mathbf{s}\right) e\left({ }^{t}(\mathbf{x}+\mathbf{r}) Z(\mathbf{x}+\mathbf{r}) / 2\right)}{\sum_{\mathbf{x} \in \mathbb{Z}^{g}} e\left(\left({ }^{(} \mathbf{x} Z \mathbf{x}\right) / 2\right)} \text { by Proposition 2.1(i) } \\
= & \Phi_{\left[\begin{array}{c}
\mathbf{r} \\
a \mathbf{s}
\end{array}\right](Z)} \\
= & \Phi_{t_{\iota\left(a^{-1}\right)}\left[\begin{array}{r}
\mathbf{r} \\
\mathbf{s}
\end{array}\right]}(Z) .
\end{aligned}
$$

Lemma 4.2. For even $N$, let $\gamma=I_{2 g}+N\left[\begin{array}{cc}A_{0} & B_{0} \\ C_{0} & D_{0}\end{array}\right] \in \Gamma(N)$ with $A_{0}, B_{0}, C_{0}, D_{0}$ $\in \operatorname{Mat}_{g}(\mathbb{Z})$. Then we have

$$
\begin{aligned}
& \Phi_{\left[\begin{array}{c}
\mathbf{r} \\
\mathbf{s}
\end{array}\right]}(\gamma(Z)) \\
= & e\left(-\frac{1}{2 N}{ }^{t}(N \mathbf{r})\left(-{ }^{t} B_{0}+N A_{0}{ }^{t} B_{0}\right)(N \mathbf{r})-\frac{1}{2 N}{ }^{t}(N \mathbf{s})\left(C_{0}+N C_{0}{ }^{t} D_{0}\right)(N \mathbf{s})\right. \\
& -\frac{1}{N}{ }^{t}(N \mathbf{r})\left(A_{0}+(N / 2)\left(A_{0}{ }^{t} D_{0}+{ }^{t} D_{0} A_{0}+B_{0}{ }^{t} C_{0}-{ }^{t} B_{0} C_{0}\right)(N \mathbf{s})\right) \Phi_{\left[\begin{array}{l}
\mathbf{r} \\
\mathbf{s}
\end{array}\right]}(Z) .
\end{aligned}
$$

Proof. We obtain from the relation ${ }^{t} \gamma J \gamma=J$ that

$$
\left[\begin{array}{cc}
* & -I_{g}-N^{t} A_{0}-N D_{0}-\left(N^{t} A_{0}\right)\left(N D_{0}\right)+\left(N^{t} C_{0}\right)\left(N B_{0}\right) \\
* & *
\end{array}\right]=\left[\begin{array}{cc}
0 & -I_{g} \\
I_{g} & 0
\end{array}\right],
$$

which gives rise to

$$
D_{0}=-{ }^{t} A_{0}-N^{t} A_{0} D_{0}+N^{t} C_{0} B_{0} .
$$

We then derive that

$$
\Phi_{\left[\begin{array}{l}
\mathbf{r} \\
\mathbf{s}
\end{array}\right]}(\gamma(Z))
$$




$$
\begin{aligned}
& =\frac{\Theta(\mathbf{0}, \gamma(Z) ; \mathbf{r}, \mathbf{s})}{\Theta(\mathbf{0}, \gamma(Z) ; \mathbf{0}, \mathbf{0})} \\
& =\frac{e\left(\left({ }^{t} \mathbf{r s}-{ }^{t} \mathbf{r}^{\prime} \mathbf{s}^{\prime}\right) / 2\right) \Theta\left(\mathbf{0}, Z ; \mathbf{r}^{\prime}, \mathbf{s}^{\prime}\right)}{\Theta(\mathbf{0}, Z ; \mathbf{0}, \mathbf{0})} \quad \text { where }\left[\begin{array}{c}
\mathbf{r}^{\prime} \\
\mathbf{s}^{\prime}
\end{array}\right]={ }^{t} \gamma\left[\begin{array}{l}
\mathbf{r} \\
\mathbf{s}
\end{array}\right],
\end{aligned}
$$

by Proposition 3.2

$$
\begin{aligned}
= & e\left(-\frac{1}{2 N}^{t}(N \mathbf{r})\left({ }^{t} B_{0}+N A_{0}{ }^{t} B_{0}\right)(N \mathbf{r})-\frac{1}{2 N}^{t}(N \mathbf{s})\left(C_{0}+N C_{0}{ }^{t} D_{0}\right)(N \mathbf{s})\right. \\
& \left.-\frac{1}{2 N}^{t}(N \mathbf{r})\left(A_{0}+{ }^{t} D_{0}+N A_{0}{ }^{t} D_{0}+N B_{0}{ }^{t} C_{0}\right)(N \mathbf{s})\right) \Phi_{\left[\begin{array}{l}
\mathbf{r}+{ }^{t} A_{0}(N \mathbf{r})+{ }^{t} C_{0}(N \mathbf{s}) \\
\mathbf{s}+{ }^{t} B_{0}(N \mathbf{r})+{ }^{t} D_{0}(N \mathbf{s})
\end{array}\right]}(Z) \\
= & e\left(-\frac{1}{2 N}^{t}(N \mathbf{r})\left(-{ }^{t} B_{0}+N A_{0}{ }^{t} B_{0}\right)(N \mathbf{r})-\frac{1}{2 N}^{t}(N \mathbf{s})\left(C_{0}+N C_{0}{ }^{t} D_{0}\right)(N \mathbf{s})\right. \\
& \left.-\frac{1}{2 N}^{t}(N \mathbf{r})\left(A_{0}-{ }^{t} D_{0}+N A_{0}{ }^{t} D_{0}+N B_{0}{ }^{t} C_{0}\right)(N \mathbf{s})\right) \Phi_{\left[\begin{array}{r}
\mathbf{r} \\
\mathbf{s}]
\end{array}\right.}(Z)
\end{aligned}
$$

by Proposition 3.1

$$
\begin{aligned}
= & e\left(-\frac{1}{2 N}^{t}(N \mathbf{r})\left(-{ }^{t} B_{0}+N A_{0}{ }^{t} B_{0}\right)(N \mathbf{r})-\frac{1}{2 N}^{t}(N \mathbf{s})\left(C_{0}+N C_{0}{ }^{t} D_{0}\right)(N \mathbf{s})\right. \\
& -\frac{1}{N}^{t}(N \mathbf{r})\left(A_{0}+(N / 2)\left(A_{0}{ }^{t} D_{0}+{ }^{t} D_{0} A_{0}+B_{0}{ }^{t} C_{0}-{ }^{t} B_{0} C_{0}\right)(N \mathbf{s})\right) \Phi_{\left[\begin{array}{l}
\mathbf{r} \\
\mathbf{s}
\end{array}\right]}(Z)
\end{aligned}
$$

by (4).

Theorem 4.3. For even $N$, let $\{m(\mathbf{r}, \mathbf{s})\}_{\mathbf{r}, \mathbf{s}}$, where $\mathbf{r}, \mathbf{s} \in(1 / N) \mathbb{Z}^{g}$ such that $\left[\begin{array}{l}\mathbf{r} \\ \mathbf{s}\end{array}\right] \notin \Sigma_{-}$, be a family of integers such that $m(\mathbf{r}, \mathbf{s})=0$ except finitely many pairs of $\mathbf{r}, \mathbf{s}$. Consider the following product

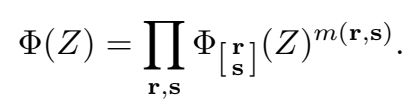

Then, $\Phi(Z)$ belongs to $\mathcal{F}_{N}$ if the family $\{m(\mathbf{r}, \mathbf{s})\}_{\mathbf{r}, \mathbf{s}}$ satisfies the condition

$$
\left\{\begin{aligned}
\sum_{\mathbf{r}, \mathbf{s}} m(\mathbf{r}, \mathbf{s})\left(N \mathbf{r}_{j}\right)\left(N \mathbf{r}_{k}\right) & \equiv \sum_{\mathbf{r}, \mathbf{s}} m(\mathbf{r}, \mathbf{s})\left(N \mathbf{s}_{j}\right)\left(N \mathbf{s}_{k}\right) \\
& \equiv 0 \quad(\bmod 2 N) \quad(1 \leq j, k \leq g), \\
\sum_{\mathbf{r}, \mathbf{s}} m(\mathbf{r}, \mathbf{s})\left(N \mathbf{r}_{j}\right)\left(N \mathbf{s}_{k}\right) & \equiv 0 \quad(\bmod N) \quad(1 \leq j, k \leq g),
\end{aligned}\right.
$$

where $\mathbf{r}=\left[\begin{array}{c}\mathbf{r}_{1} \\ \vdots \\ \mathbf{r}_{g}\end{array}\right]$ and $\mathbf{s}=\left[\begin{array}{c}\mathbf{s}_{1} \\ \vdots \\ \mathbf{s}_{g}\end{array}\right]$.

Proof. If $\gamma \in \Gamma(N)$, then we have

(6) $\Phi(\gamma(Z))=\prod_{\mathbf{r}, \mathbf{s}}\left(\frac{\Theta(\mathbf{0}, \gamma(Z) ; \mathbf{r}, \mathbf{s})}{\Theta(\mathbf{0}, \gamma(Z) ; \mathbf{0}, \mathbf{0})}\right)^{m(\mathbf{r}, \mathbf{s})}$ 


$$
\begin{aligned}
= & e\left(-\frac{1}{2 N} \sum_{\mathbf{r}, \mathbf{s}} m(\mathbf{r}, \mathbf{s})^{t}(N \mathbf{r})\left(-{ }^{t} B_{0}+N A_{0}{ }^{t} B_{0}\right)(N \mathbf{r})\right. \\
& -\frac{1}{2 N} \sum_{\mathbf{r}, \mathbf{s}} m(\mathbf{r}, \mathbf{s})^{t}(N \mathbf{s})\left(C_{0}+N C_{0}{ }^{t} D_{0}\right)(N \mathbf{s}) \\
& -\frac{1}{N} \sum_{\mathbf{r}, \mathbf{s}} m(\mathbf{r}, \mathbf{s})^{t}(N \mathbf{r})\left(A_{0}+\right. \\
& \left.\left.(N / 2)\left(A_{0}{ }^{t} D_{0}+{ }^{t} D_{0} A_{0}+B_{0}{ }^{t} C_{0}-{ }^{t} B_{0} C_{0}\right)\right)(N \mathbf{s})\right) \Phi(Z),
\end{aligned}
$$

where $\left[\begin{array}{cc}A_{0} & B_{0} \\ C_{0} & D_{0}\end{array}\right]=\frac{1}{N}\left(\gamma-I_{2 g}\right) \in \operatorname{Mat}_{2 g}(\mathbb{Z})$, by Lemma 4.2 .

Now, for every pair of integers $j, k$ with $1 \leq j, k \leq g$ let $E_{j k}$ be the $g \times g$ matrix whose entries are all zeros except for the $(j, k)$ th entry which is 1 . One can then easily see that $\operatorname{Mat}_{g}(\mathbb{Z})$ is generated by $E_{j k}(1 \leq j, k \leq g)$ as $\mathbb{Z}$-module, and if $\mathbf{u}=\left[\begin{array}{c}\mathbf{u}_{1} \\ \vdots \\ \mathbf{u}_{g}\end{array}\right], \mathbf{v}=\left[\begin{array}{c}\mathbf{v}_{1} \\ \vdots \\ \mathbf{v}_{g}\end{array}\right] \in \mathbb{Z}^{g}$, then ${ }^{t} \mathbf{u} E_{j k} \mathbf{v}=\mathbf{u}_{j} \mathbf{v}_{k}$.

Assume first that the family $\{m(\mathbf{r}, \mathbf{s})\}_{\mathbf{r}, \mathbf{s}}$ satisfies the condition (5). Then the above observation leads to $\Phi(\gamma(\tau))=\Phi(\tau)$. On the other hand, since $\Phi(Z)$ belongs to $\mathcal{F}_{2 N^{2}}$, its Fourier coefficients of $\Phi(Z)$ lie in $\mathbb{Q}\left(\zeta_{2 N^{2}}\right)$. However, at this stage we have to show that the coefficients actually lie in $\mathbb{Q}\left(\zeta_{N}\right)$. To this end, let $a$ be an integer such that $a \equiv 1(\bmod N)$, which can be written as $a=1+c N$ for some integer $c$. Regarding $\iota$ as a map on $\left(\mathbb{Z} / 2 N^{2} \mathbb{Z}\right)^{\times}$we get that

$$
\begin{aligned}
\Phi(Z)^{\iota(a)^{-1}} & =\prod_{\mathbf{r}, \mathbf{s}}\left(\Phi_{\left[\begin{array}{c}
\mathbf{r} \\
\mathbf{s}
\end{array}\right]}(Z)^{\iota(a)^{-1}}\right)^{m(\mathbf{r}, \mathbf{s})} \\
& =\prod_{\mathbf{r}, \mathbf{s}} \Phi_{\left[\begin{array}{c}
\mathbf{r} \\
a \mathbf{s}
\end{array}\right]}(Z)^{m(\mathbf{r}, \mathbf{s})} \quad \text { by Lemma } 4.1 \\
& =\prod_{\mathbf{r}, \mathbf{s}} \Phi_{\left[\begin{array}{c}
\mathbf{r}+c N \mathbf{s} \\
\mathbf{r}
\end{array}\right]}(Z)^{m(\mathbf{r}, \mathbf{s})} \\
& =\prod_{\mathbf{r}, \mathbf{s}}\left(e\left({ }^{t} \mathbf{r} c N \mathbf{s}\right) \Phi_{\left[\begin{array}{l}
\mathbf{r} \\
\mathbf{s}
\end{array}\right]}(Z)\right)^{m(\mathbf{r}, \mathbf{s}) \quad \text { by Proposition } 3.1} \\
& =e\left(\frac{c}{N} \sum_{\mathbf{r}, \mathbf{s}} m(\mathbf{r}, \mathbf{s})^{t}(N \mathbf{r})(N \mathbf{s})\right) \Phi(Z) \\
& =e\left(\frac{c}{N} \sum_{j=1}^{g} \sum_{\mathbf{r}, \mathbf{s}} m(\mathbf{r}, \mathbf{s})\left(N \mathbf{r}_{j}\right)\left(N \mathbf{s}_{j}\right)\right) \Phi(Z) \\
& =\Phi(Z) \quad \text { by the condition }(5),
\end{aligned}
$$

which ensures that Fourier coefficients of $\Phi(Z)$ lie in $\mathbb{Q}\left(\zeta_{N}\right)$ as desired. Therefore we conclude that $\Phi(Z)$ is in $\mathcal{F}_{N}$. 


\section{Family of theta constants}

Let $N$ be an even positive integer and $\mathbf{r}, \mathbf{s} \in(1 / N) \mathbb{Z}^{g}(g \geq 2)$. By Theorem $4.3, \Phi_{\left[\begin{array}{l}\mathbf{r} \\ \mathbf{s}\end{array}\right]}(Z)^{2 N}$ belongs to $\mathcal{F}_{N}$. In this section we shall show that a subgroup of $\operatorname{GSp}_{2 g}(\mathbb{Z} / N \mathbb{Z})$ has a natural action on the family $\left\{\Phi_{\left[\begin{array}{l}\mathbf{r} \\ \mathbf{s}\end{array}\right]}(Z)^{2 N^{2}}\right\}_{\mathbf{r}, \mathbf{s} \in(1 / N) \mathbb{Z}^{g} / \mathbb{Z}^{g}}$.

Lemma 5.1. Let $M$ and $\ell$ be divisors of $N$. If $\mathbf{r} \in(1 / M) \mathbb{Z}^{g}$, then $\Phi_{\left[\begin{array}{l}\mathbf{r} \\ \mathbf{s}\end{array}\right]}(Z)^{\ell}$ is determined by $\mathbf{r}\left(\bmod \mathbb{Z}^{g}\right)$ and $\mathbf{s}\left(\bmod (M / \operatorname{gcd}(M, \ell)) \mathbb{Z}^{g}\right)$.

Proof. If $\mathbf{a} \in \mathbb{Z}^{g}$ and $\mathbf{b} \in(M / \operatorname{gcd}(M, \ell)) \mathbb{Z}^{g}$, then we see by Proposition 3.1 that

$$
\Phi_{\left[\begin{array}{l}
\mathbf{r}+\mathbf{a} \\
\mathbf{s}+\mathbf{b}
\end{array}\right]}(Z)^{\ell}=\left(e\left({ }^{t} \mathbf{r b}\right) \Phi_{\left[\begin{array}{l}
\mathbf{r} \\
\mathbf{s}
\end{array}\right]}(Z)\right)^{\ell}=\Phi_{\left[\begin{array}{l}
\mathbf{r} \\
\mathbf{s}
\end{array}\right]}(Z)^{\ell} .
$$

Let

$$
\begin{aligned}
S_{N} & =\left\{\left[\begin{array}{ll}
A & B \\
C & D
\end{array}\right] \in \mathrm{Sp}_{2 g}(\mathbb{Z} / N \mathbb{Z}) \mid\left\{{ }^{t} A C\right\} \equiv\left\{{ }^{t} B D\right\} \equiv \mathbf{0} \quad(\bmod 2)\right\}, \\
G_{N} & =\left\langle\iota\left((\mathbb{Z} / N \mathbb{Z})^{\times}\right), S_{N}\right\rangle
\end{aligned}
$$

which are subgroups of $\mathrm{GSp}_{2 g}(\mathbb{Z} / N \mathbb{Z})[12, \S 27.6]$. One can then readily show that

$$
G_{N}=\left\{\left[\begin{array}{cc}
A & B \\
C & D
\end{array}\right] \in \operatorname{GSp}_{2 g}(\mathbb{Z} / N \mathbb{Z}) \mid\left\{{ }^{t} A C\right\} \equiv\left\{{ }^{t} B D\right\} \equiv \mathbf{0} \quad(\bmod 2)\right\} .
$$

Theorem 5.2. If $\alpha \in G_{N}$, then we have

$$
\left(\Phi_{\left[\begin{array}{l}
\mathbf{r} \\
\mathbf{s}
\end{array}\right]}(Z)^{2 N^{2}}\right)^{\alpha}=\Phi_{t_{\alpha}\left[\begin{array}{l}
\mathbf{r} \\
\mathbf{s}
\end{array}\right]}(Z)^{2 N^{2}}
$$

Proof. If $\alpha \in \iota\left((\mathbb{Z} / N \mathbb{Z})^{\times}\right)$, then the assertion follows from Lemmas 4.1 and 5.1. Let $\alpha \in S_{N}$ with a lifting $\alpha_{0}=\left[\begin{array}{ll}A_{0} & B_{0} \\ C_{0} & D_{0}\end{array}\right]$ to $\operatorname{Sp}_{2 g}(\mathbb{Z})$. Note that since $N$ is even, $\left\{{ }^{t} A_{0} C_{0}\right\},\left\{{ }^{t} B_{0} D_{0}\right\} \in 2 \mathbb{Z}^{g}$. Thus we derive that

$$
\left(\Phi_{\left[\begin{array}{c}
\mathbf{r} \\
\mathbf{s}
\end{array}\right]}(Z)^{2 N^{2}}\right)^{\alpha}
$$

$=\left(\Phi_{\left[\begin{array}{l}\mathbf{r} \\ \mathbf{s}\end{array}\right]}(Z)^{2 N^{2}}\right)^{\alpha_{0}} \quad$ by Proposition 2.1(iii)

$=\Phi_{\left[\begin{array}{l}\mathbf{r} \\ \mathbf{s}\end{array}\right]}(Z)^{2 N^{2}} \circ \alpha_{0}$ by Proposition 2.1(ii)

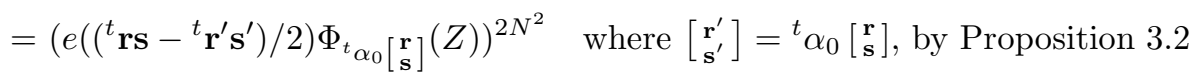

$=\Phi_{t_{\alpha}\left[\begin{array}{c}\mathbf{r} \\ \mathbf{s}\end{array}\right]}(Z)^{2 N^{2}} \quad$ by Lemma 5.1 .

Since $G_{N}=\left\langle\iota\left((\mathbb{Z} / N \mathbb{Z})^{\times}\right), S_{N}\right\rangle$, we get the theorem.

Remark 5.3. (i) This theorem tells us that the group $G_{N}$ acts on the family $\left\{\Phi_{\left[\begin{array}{l}\mathbf{r} \\ \mathbf{s}\end{array}\right]}(Z)^{2 N^{2}}\right\}_{\mathbf{r}, \mathbf{s} \in(1 / N) \mathbb{Z}^{g} / \mathbb{Z}^{g}}$ in a natural way.

(ii) For later use we consider the case where $\mathbf{r}, \mathbf{s} \in(1 / M) \mathbb{Z}^{g}$ for an odd positive integer $M$. Then $\Phi_{\left[\begin{array}{l}\mathbf{r} \\ \mathbf{s}\end{array}\right]}(Z)$ (respectively, $\Phi_{\left[\begin{array}{l}\mathbf{r} \\ \mathbf{s}\end{array}\right]}(Z)^{M}$ ) is of level $2 M^{2}$ 
(respectively, $2 M$ ) by Theorem 4.3. As in the proof of Theorem 5.2 one can show in a similar way that if $\alpha \in G_{2 M}$, then

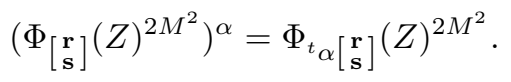

Now, let $\alpha \in G_{2 M^{2}}$ with $a=\nu(\alpha)$ and set $\gamma=\iota(a) \alpha \in S_{2 M^{2}}$. Then we achieve that

$$
\begin{aligned}
& \Phi_{\left[\begin{array}{l}
\mathbf{r} \\
\mathbf{s}
\end{array}\right]}(Z)^{\alpha} \\
= & \Phi_{\left[\begin{array}{l}
\mathbf{r} \\
\mathbf{s}
\end{array}\right]}(Z)^{\iota(a)^{-1} \gamma} \\
= & \Phi_{\left[\begin{array}{c}
\mathbf{r} \\
a
\end{array}\right]}(Z)^{\gamma} \text { by Lemmas } 4.1 \text { and } 5.1 \\
= & e\left(\left({ }^{t} \mathbf{r} a \mathbf{s}-{ }^{t} \mathbf{r}^{\prime} \mathbf{s}^{\prime}\right) / 2\right) \Phi_{\left[\begin{array}{l}
\mathbf{r}^{\prime} \\
\mathbf{s}^{\prime}
\end{array}\right]}(Z) \quad \text { where }\left[\begin{array}{l}
\mathbf{r}^{\prime} \\
\mathbf{s}^{\prime}
\end{array}\right]={ }^{t} \gamma\left[\begin{array}{l}
\mathbf{r} \\
a \mathbf{s}
\end{array}\right]={ }^{t} \gamma^{t} \iota\left(a^{-1}\right)\left[\begin{array}{l}
\mathbf{r} \\
\mathbf{s}
\end{array}\right]={ }^{t} \alpha\left[\begin{array}{l}
\mathbf{r} \\
\mathbf{s}
\end{array}\right],
\end{aligned}
$$

by Proposition 3.2 and Lemma 5.1

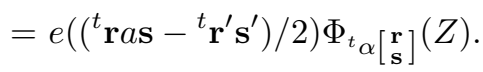

Lemma 5.4. Let $Z_{0} \in \mathbb{H}_{g}$. If $\Theta\left(\mathbf{0}, Z_{0} ; \mathbf{0}, \mathbf{0}\right)$ is nonzero, then we have

$$
\overline{\Phi_{\left[\begin{array}{l}
\mathbf{r} \\
\mathbf{s}
\end{array}\right]}\left(Z_{0}\right)}=\Phi_{\left[\begin{array}{c}
\mathbf{r} \\
-\mathbf{s}
\end{array}\right]}\left(-\bar{Z}_{0}\right),
$$

where the bar indicates the usual complex conjugation.

Proof. Consider the expansion $\Phi_{\left[\begin{array}{l}\mathbf{r} \\ \mathbf{s}\end{array}\right]}(Z)=\sum_{\xi} c(\xi) e\left(\operatorname{tr}(\xi Z) / 2 N^{2}\right) / \Theta(\mathbf{0}, Z ; \mathbf{0}, \mathbf{0})$ with $c(\xi) \in \mathbb{Q}\left(\zeta_{2 N^{2}}\right)$, where $\xi$ runs over all $g \times g$ positive semi-definite symmetric matrices over half integers with integral diagonal entries. Note that $\Theta(\mathbf{0}, Z ; \mathbf{0}, \mathbf{0})$ has rational Fourier coefficients. Then we obtain that

$$
\begin{aligned}
& \overline{\Phi_{\left[\begin{array}{l}
\mathbf{r} \\
\mathbf{s}
\end{array}\right]}\left(Z_{0}\right)} \\
= & \sum_{\xi} \overline{c(\xi)} e\left(\operatorname{tr}\left(\xi\left(-\bar{Z}_{0}\right)\right) / 2 N^{2}\right) / \Theta\left(\mathbf{0},-\bar{Z}_{0} ; \mathbf{0}, \mathbf{0}\right) \quad \text { because } \overline{e(z)}=e(-\bar{z})(z \in \mathbb{C}) \\
= & \sum_{\xi} c(\xi)^{\rho(-1)} e\left(\operatorname{tr}\left(\xi\left(-\bar{Z}_{0}\right)\right) / 2 N^{2}\right) / \Theta\left(\mathbf{0},-\bar{Z}_{0} ; \mathbf{0}, \mathbf{0}\right) \quad \text { where } \rho(-1) \text { is the } \\
& \text { endomorphism of } \mathbb{Q}\left(\zeta_{2 N^{2}}\right) \text { induced from } \zeta_{2 N^{2}} \mapsto \zeta_{2 N^{2}}^{-1}=\bar{\zeta}_{2 N^{2}} \\
= & \left(\sum_{\xi} c(\xi) e\left(\operatorname{tr}(\xi Z) / 2 N^{2}\right) / \Theta(\mathbf{0}, Z ; \mathbf{0}, \mathbf{0})\right)^{\iota(-1)^{-1}}\left(-\bar{Z}_{0}\right) \quad \text { by Proposition } 2.1(\mathrm{i}) \\
= & \Phi_{\left[\begin{array}{l}
\mathbf{r} \\
\mathbf{s}
\end{array}\right]}(Z)^{\iota(-1)^{-1}}\left(-\bar{Z}_{0}\right) \\
= & \Phi_{\left[\begin{array}{c}
\mathbf{r} \\
-\mathbf{s}
\end{array}\right]}\left(-\bar{Z}_{0}\right) \quad \text { by Lemma 4.1. }
\end{aligned}
$$

\section{Shimura's reciprocity law}

We shall briefly explain Shimura's reciprocity law which connects the theory of Siegel modular functions with class field theory. 
Let $g$ be a positive integer and $K$ be a CM-field with $[K: \mathbb{Q}]=2 g$, that is, a totally imaginary quadratic extension of a totally real algebraic number field. Fix a set $\left\{\varphi_{1}, \ldots, \varphi_{g}\right\}$ of $g$ embeddings $K \rightarrow \mathbb{C}$ such that no two of them are complex conjugate. One can take an element $\xi \in K$ satisfying

(i) $\xi$ is purely imaginary,

(ii) $-\xi^{2}$ is totally positive,

(iii) $\operatorname{Im}\left(\xi^{\varphi_{k}}\right)>0$ for all $k=1, \ldots, g$

(iv) $\operatorname{Tr}_{K / \mathbb{Q}}(\xi x) \in \mathbb{Z}$ for all $x \in \mathcal{O}_{K}$

([12, p.43]). Define a map $\Phi: K \rightarrow \mathbb{C}^{g}$ by $\Phi(x)=\left[\begin{array}{c}x^{\varphi_{1}} \\ \vdots \\ x^{\varphi_{g}}\end{array}\right]$, and let $L=\{\Phi(x) \in$ $\left.\mathbb{C}^{g} \mid x \in \mathcal{O}_{K}\right\}$ which forms a lattice in $\mathbb{C}^{g}$. Then we have an $\mathbb{R}$-bilinear map $E: \mathbb{C}^{g} \times \mathbb{C}^{g} \rightarrow \mathbb{R}$ defined by

$$
E(\mathbf{u}, \mathbf{v})=\sum_{k=1}^{g} \xi^{\varphi_{k}}\left(u_{k} \bar{v}_{k}-\bar{u}_{k} v_{k}\right) \quad\left(\mathbf{u}=\left[\begin{array}{c}
u_{1} \\
\vdots \\
u_{g}
\end{array}\right], \mathbf{v}=\left[\begin{array}{c}
v_{1} \\
\vdots \\
v_{g}
\end{array}\right]\right)
$$

And $E$ gives a non-degenerate Riemann form on the complex torus $\mathbb{C}^{g} / L$ satisfying $E(\Phi(x), \Phi(y))=\operatorname{Tr}_{K / \mathbb{Q}}(\xi x \bar{y}) \in \mathbb{Z}$ for all $x, y \in \mathcal{O}_{K}[12$, p. 44], which makes it an abelian variety. Hence one can find a positive integer $\mu$, a diagonal $\operatorname{matrix} \mathcal{E}=\left[\begin{array}{ccc}\varepsilon_{1} & & \\ & \ddots & \\ & & \varepsilon_{g}\end{array}\right] \in \operatorname{Mat}_{g}(\mathbb{Z})$ with $\varepsilon_{1}=1$ and $\varepsilon_{k} \mid \varepsilon_{k+1}(k=1, \ldots, g-1)$, and a complex $g \times 2 g$ matrix $\Omega=\left[\omega_{1} \omega_{2}\right]$ with $\omega_{1}, \omega_{2} \in \operatorname{Mat}_{g}(\mathbb{C})$ such that

(i) $E(\Omega \mathbf{x}, \Omega \mathbf{y})=\mu^{t} \mathbf{x} J \mathbf{y}$ for all $\mathbf{x}, \mathbf{y} \in \mathbb{R}^{2 g}$,

(ii) $L=\left\{\Omega\left[\begin{array}{l}\mathbf{u} \\ \mathbf{v}\end{array}\right] \mid \mathbf{u} \in \mathbb{Z}^{g}, \mathbf{v} \in \mathcal{E} \mathbb{Z}^{g}\right\}$

[10, p. 675]. It is well-known that $Z_{0}=\omega_{2}^{-1} \omega_{1}$ lies in $\mathbb{H}_{g}$. Let $K^{*}$ be the reflex field of $K$, namely $K^{*}=\mathbb{Q}\left(\sum_{k=1}^{g} x^{\varphi_{k}} \mid x \in K\right)$. As a consequence of the main theorem of complex multiplication we have the following proposition.

Proposition 6.1. If $f$ is an element of $\mathcal{F}_{N}$ which is finite at $Z_{0}$, then the special value $f\left(Z_{0}\right)$ belongs to the maximal abelian extension of $K^{*}$.

Proof. See [12, Theorem 26.6].

For simplicity and later use, we assume that $\mathcal{E}=I_{g}$. We define, as a representation map, a ring homomorphism $h: K \rightarrow$ Mat $_{2 g}(\mathbb{Q})$ as follows: Fix $\xi_{1}, \xi_{2}, \ldots, \xi_{2 g} \in K$ such that $\Omega=\left[\begin{array}{llll}\Phi\left(\xi_{1}\right) & \Phi\left(\xi_{2}\right) & \cdots & \Phi\left(\xi_{2 g}\right)\end{array}\right]$ and let $x \in K$. If $x \xi_{j}=\sum_{k=1}^{2 g} r_{j k} \xi_{k}$ with $r_{j k} \in \mathbb{Q}(j=1, \ldots, g)$, then we define

$$
h(x)=\left[r_{j k}\right]_{1 \leq j, k \leq 2 g} .
$$

Since $L=\Phi\left(\mathcal{O}_{K}\right)=\Omega \mathbb{Z}^{2 g}=\mathbb{Z} \Phi\left(\xi_{1}\right)+\cdots+\mathbb{Z} \Phi\left(\xi_{2 g}\right)$ by the assumption $\mathcal{E}=I_{g}$, the set $\left\{\xi_{1}, \ldots, \xi_{2 g}\right\}$ forms a basis of $\mathcal{O}_{K}$ as $\mathbb{Z}$-module. Hence, if $x \in \mathcal{O}_{K}$, then $h(x) \in \operatorname{Mat}_{2 g}(\mathbb{Z})$. 
On the other hand, we take a Galois extension $K^{\prime}$ of $\mathbb{Q}$ containing $K$, and extend $\varphi_{k}(k=1, \ldots, g)$ to an element of $\operatorname{Gal}\left(K^{\prime} / \mathbb{Q}\right)$. We then define a homomorphism $\varphi^{*}:\left(K^{*}\right)^{\times} \rightarrow K^{\times}$by

$$
\varphi^{*}(x)=\prod_{k=1}^{g} x^{\varphi_{k}^{-1}} \quad\left(x \in\left(K^{*}\right)^{\times}\right) .
$$

Proposition 6.2 (Shimura's reciprocity law). Let $f$ be as in Proposition 6.1. Take a positive integer $M$ such that $N \mid M$ and $f\left(Z_{0}\right) \in K_{(M)}^{*}$. Let $x$ be an element of $\mathcal{O}_{K^{*}}$ which is prime to $M$. Considering $h\left(\varphi^{*}(x)\right)$ as an element of $\mathrm{GSp}_{2 g}(\mathbb{Z} / M \mathbb{Z})$ we have

$$
f\left(Z_{0}\right)^{\left(\frac{K_{(M)}^{*} / K^{*}}{(x)}\right)}=f^{h\left(\varphi^{*}(x)\right)}\left(Z_{0}\right)
$$

where $\left(\frac{K_{(M)}^{*} / K^{*}}{\text {. }}\right)$ is the Artin reciprocity map.

Proof. See [12, Theorem 26.8] or [8, §2.7].

Remark 6.3. Assume further $x \equiv 1(\bmod N)$. It is obvious that $\varphi^{*}(x) \equiv 1$ $(\bmod N)$, which yields $h\left(\varphi^{*}(x)\right) \equiv I_{2 g}(\bmod N)$. Hence we get by Proposition $6.2 f\left(Z_{0}\right)^{\left(\frac{K_{(M)}^{*} / K^{*}}{(x)}\right)}=f^{h\left(\varphi^{*}(x)\right)}\left(Z_{0}\right)=f\left(Z_{0}\right)$. This means that $f\left(Z_{0}\right)$ lies in $K_{(N)}^{*}$.

\section{Construction of class fields}

Let $K=\mathbb{Q}(\zeta)$ with $\zeta=\zeta_{5}$, which is a CM-field of degree $2 g=[K: \mathbb{Q}]=4$. In this section we shall examine the subfield of $K_{(2 p)}$ for an odd prime $p$ which is generated by the special value of $\Phi\left[\begin{array}{c}1 / p \\ 0 \\ 0 \\ 0\end{array}\right](Z)^{2 p^{2}}$ by using Shimura's reciprocity law.

Fix a set of two embeddings $\left\{\varphi_{1}, \varphi_{2}\right\}$, where $\varphi_{1}$ and $\varphi_{2}$ are defined by

$$
\zeta^{\varphi_{1}}=\zeta \text { and } \zeta^{\varphi_{2}}=\zeta^{2} \text {. }
$$

If we set $\xi=\left(\zeta-\zeta^{4}\right) / 5$, then one can readily check that $\xi$ satisfies the conditions (i) $\sim$ (iv) in the beginning of $\S 6$. Let $\Phi: K \rightarrow \mathbb{C}^{2}$ be the map given by $\Phi(x)=$ $\left[\begin{array}{l}x^{\varphi_{1}} \\ x^{\varphi_{2}}\end{array}\right]$ and $L=\Phi\left(\mathcal{O}_{K}\right)$. We have an $\mathbb{R}$-bilinear map $E: \mathbb{C}^{2} \times \mathbb{C}^{2} \rightarrow \mathbb{R}$ defined

$$
E(\mathbf{u}, \mathbf{v})=\xi^{\varphi_{1}}\left(u_{1} \bar{v}_{1}-\bar{u}_{1} v_{1}\right)+\xi^{\varphi_{2}}\left(u_{2} \bar{v}_{2}-\bar{u}_{2} v_{2}\right) \quad\left(\mathbf{u}=\left[\begin{array}{l}
u_{1} \\
u_{2}
\end{array}\right], \mathbf{v}=\left[\begin{array}{l}
v_{1} \\
v_{2}
\end{array}\right]\right),
$$

which induces a non-degenerate Riemann form on $\mathbb{C}^{2} / L$ by $\S 6$. Set

$$
\xi_{1}=\zeta^{2}, \xi_{2}=\zeta^{4}, \xi_{3}=\zeta, \xi_{4}=\zeta+\zeta^{3},
$$


and

$$
\Omega=\left[\begin{array}{llll}
\Phi\left(\xi_{1}\right) & \Phi\left(\xi_{2}\right) & \Phi\left(\xi_{3}\right) & \Phi\left(\xi_{4}\right)
\end{array}\right]=\left[\begin{array}{cccc}
\zeta^{2} & \zeta^{4} & \zeta & \zeta+\zeta^{3} \\
\zeta^{4} & \zeta^{3} & \zeta^{2} & \zeta^{2}+\zeta
\end{array}\right] .
$$

One can readily verify that $\left[E\left(\Phi\left(\xi_{j}\right), \Phi\left(\xi_{k}\right)\right)\right]_{1 \leq j, k \leq 4}=J$, from which it follows that $E(\Omega \mathbf{x}, \Omega \mathbf{y})={ }^{t} \mathbf{x} J \mathbf{y}$ for all $\mathbf{x}, \mathbf{y} \in \mathbb{R}^{4}$ because $E$ is $\mathbb{R}$-bilinear. Furthermore, since $\mathcal{O}_{K}=\mathbb{Z}[\zeta]=\xi_{1} \mathbb{Z}+\xi_{2} \mathbb{Z}+\xi_{3} \mathbb{Z}+\xi_{4} \mathbb{Z}$, we get $L=\Phi\left(\mathcal{O}_{K}\right)=\Omega \mathbb{Z}^{4}$. (So, $\mu=1$ and $\mathcal{E}=I_{2}$ in $\S 6$.) Then we obtain a CM-point

$$
Z_{0}=\left[\begin{array}{ll}
\Phi\left(\xi_{3}\right) & \Phi\left(\xi_{4}\right)
\end{array}\right]^{-1}\left[\begin{array}{ll}
\Phi\left(\xi_{1}\right) & \Phi\left(\xi_{2}\right)
\end{array}\right]=\left[\begin{array}{cc}
\zeta & \zeta+\zeta^{3} \\
\zeta^{2} & \zeta^{2}+\zeta
\end{array}\right]^{-1}\left[\begin{array}{cc}
\zeta^{2} & \zeta^{4} \\
\zeta^{4} & \zeta^{3}
\end{array}\right] \quad\left(\in \mathfrak{H}_{2}\right)
$$

Now we define a ring homomorphism $h: K \rightarrow \operatorname{Mat}_{4}(\mathbb{Q})$ by the relation

$$
x\left[\begin{array}{c}
\xi_{1} \\
\vdots \\
\xi_{4}
\end{array}\right]=h(x)\left[\begin{array}{c}
\xi_{1} \\
\vdots \\
\xi_{4}
\end{array}\right] \quad(x \in K)
$$

Note that the reflex field $K^{*}$ of $K$ is the same as $K$. We further define an endomorphism $\varphi^{*}$ of $K^{\times}$by

$$
\varphi^{*}(x)=x^{\varphi_{1}^{-1}} x^{\varphi_{2}^{-1}} \quad\left(x \in K^{\times}\right) .
$$

Assume that $p$ is an odd prime.

Lemma 7.1. Let $\mathbf{r}, \mathbf{s} \in(1 / p) \mathbb{Z}^{2}$.

(i) $\Theta\left(\mathbf{0}, Z_{0} ; \mathbf{0}, \mathbf{0}\right)$ is nonzero.

(ii) $2 \Phi_{\left[\begin{array}{l}\mathbf{r} \\ \mathbf{s}\end{array}\right]}\left(Z_{0}\right)$ is an algebraic integer.

(iii) $\Phi_{\left[\begin{array}{l}\mathbf{r} \\ \mathbf{s}\end{array}\right]}\left(Z_{0}\right) \in K_{\left(2 p^{2}\right)}$ and $\Phi_{\left[\begin{array}{l}\mathbf{r} \\ \mathbf{s}\end{array}\right]}\left(Z_{0}\right)^{p} \in K_{(2 p)}$.

(iv) If $\mathbf{r}=\left[\begin{array}{l}r_{1} \\ r_{2}\end{array}\right]$ and $\mathbf{s}= \pm\left[\begin{array}{c}r_{1}-r_{2} \\ -r_{1}\end{array}\right]$ for $r_{1}, r_{2} \in(1 / p) \mathbb{Z}$, then $e\left(-{ }^{t} \mathbf{r s} / 2\right) \Phi_{\left[\begin{array}{l}\mathbf{r} \\ \mathbf{s}\end{array}\right]}\left(Z_{0}\right)$ is real.

Proof. (i) See [1, p. 784].

(ii) See [5, Proposition 2].

(iii) This is immediate from Remarks 5.3(ii) and 6.3.

(iv) We observe that

$$
\bar{\Omega}=\left[\begin{array}{cccc}
\zeta^{3} & \zeta & \zeta^{4} & \zeta^{4}+\zeta^{2} \\
\zeta & \zeta^{2} & \zeta^{3} & \zeta^{3}+\zeta^{4}
\end{array}\right]=\Omega \alpha \quad \text { where } \alpha=\left[\begin{array}{cccc}
0 & 0 & 0 & 1 \\
0 & 0 & 1 & 1 \\
-1 & 1 & 0 & 0 \\
1 & 0 & 0 & 0
\end{array}\right]
$$

Let $\Omega=\left[\begin{array}{ll}\omega_{1} & \omega_{2}\end{array}\right]$ and $\alpha=\left[\begin{array}{ll}A & B \\ C & D\end{array}\right]$ with $2 \times 2$ matrices $\omega_{1}, \omega_{2}, A, B, C, D$. Then we derive that

$$
\begin{aligned}
-\bar{Z}_{0} & =-{ }^{t} \bar{Z}_{0} \quad \text { since } Z_{0}={ }^{t} Z_{0} \\
& =-{ }^{t} \bar{\omega}_{1}{ }^{t} \bar{\omega}_{2}^{-1} \\
& =-\left({ }^{t} A^{t} \omega_{1}+{ }^{t} C^{t} \omega_{2}\right)\left({ }^{t} B^{t} \omega_{1}+{ }^{t} D^{t} \omega_{2}\right)^{-1} \quad \text { by }(7)
\end{aligned}
$$




$$
\begin{aligned}
& =-\left({ }^{t} A^{t} \omega_{1}+{ }^{t} C^{t} \omega_{2}\right)^{t} \omega_{2}^{-1 t} \omega_{2}\left({ }^{t} B^{t} \omega_{1}+{ }^{t} D^{t} \omega_{2}\right)^{-1} \\
& =-\left({ }^{t} A^{t} \omega_{1}{ }^{t} \omega_{2}^{-1}+{ }^{t} C\right)\left({ }^{t} B^{t} \omega_{1}{ }^{t} \omega_{2}^{-1}+{ }^{t} D\right)^{-1} \\
& =-\left({ }^{t} A Z_{0}+{ }^{t} C\right)\left({ }^{t} B^{t} Z_{0}+{ }^{t} D\right)^{-1} \quad \text { because } Z_{0}={ }^{t} Z_{0} \\
& =\beta\left(Z_{0}\right) \quad \text { where } \beta=\left[\begin{array}{cc}
-{ }^{t} A & -{ }^{t} C \\
{ }^{t} B & { }^{t} D
\end{array}\right]=\left[\begin{array}{cccc}
0 & 0 & 1 & -1 \\
0 & 0 & -1 & 0 \\
0 & 1 & 0 & 0 \\
1 & 1 & 0 & 0
\end{array}\right] .
\end{aligned}
$$

One can readily check that $\beta \in \mathrm{Sp}_{4}(\mathbb{Z})$. Hence we achieve that

$$
\begin{aligned}
& \overline{e\left(-{ }^{t} \mathbf{r s} / 2\right) \Phi_{\left[\begin{array}{l}
\mathbf{r} \\
\mathbf{s}
\end{array}\right]}\left(Z_{0}\right)} \\
= & e\left({ }^{t} \mathbf{r s} / 2\right) \Phi_{\left[\begin{array}{l}
\mathbf{r} \\
-\mathbf{s}
\end{array}\right]}\left(-\bar{Z}_{0}\right) \quad \text { by Lemma } 5.4 \\
= & e\left({ }^{t} \mathbf{r s} / 2\right) \Phi_{\left[\begin{array}{l}
\mathbf{r} \\
-\mathbf{s}
\end{array}\right]}\left(\beta\left(Z_{0}\right)\right) \quad \text { by }(8) \\
= & e\left({ }^{t} \mathbf{r s} / 2\right) e\left(\left({ }^{t} \mathbf{r}(-\mathbf{s})-{ }^{t} \mathbf{r}^{\prime} \mathbf{s}^{\prime}\right) / 2\right) \Phi_{t \beta\left[\begin{array}{l}
{ }_{-\mathbf{s}} \\
\mathbf{r}
\end{array}\right]}\left(Z_{0}\right) \quad \text { where }\left[\begin{array}{c}
\mathbf{r}^{\prime} \\
\mathbf{s}^{\prime}
\end{array}\right]={ }^{t} \beta\left[\begin{array}{c}
\mathbf{r} \\
-\mathbf{s}
\end{array}\right], \\
& \text { by Remark } 5.3(\mathrm{ii})
\end{aligned}
$$$$
\begin{aligned}
& =\left\{\begin{array}{c}
e\left(\left(-r_{1}^{2}+2 r_{1} r_{2}\right) / 2\right) \Phi\left[\begin{array}{c}
r_{1} \\
r_{2} \\
r_{1}-r_{2} \\
-r_{1}
\end{array}\right]\left(Z_{0}\right) \quad \text { if } \mathbf{s}=\left[\begin{array}{c}
r_{1}-r_{2} \\
-r_{1}
\end{array}\right], \\
e\left(\left(r_{1}^{2}-2 r_{1} r_{2}\right) / 2\right) \Phi\left[\begin{array}{c}
-r_{1} \\
-r_{2} \\
r_{1}-r_{2}
\end{array}\right]
\end{array} \quad \text { if } \mathbf{s}=\left[\begin{array}{c}
-r_{1}+r_{2} \\
r_{1}
\end{array}\right],\right. \\
& =e\left(-{ }^{t} \mathbf{r s} / 2\right) \Phi_{\left[\begin{array}{l}
\mathbf{r} \\
\mathbf{s}
\end{array}\right]}\left(Z_{0}\right) \text { by }(3),
\end{aligned}
$$

which ensures that $e\left(-{ }^{t} \mathbf{r s} / 2\right) \Phi_{\left[\begin{array}{l}\mathbf{r} \\ \mathbf{r}\end{array}\right]}\left(Z_{0}\right)$ is real.

Now, we shall investigate the field $K\left(z^{2 p^{2}}\right)$ with

$$
z=\Phi\left[\begin{array}{c}
1 / p \\
0 \\
0 \\
0
\end{array}\right]\left(Z_{0}\right)
$$

Note that $z \in K_{\left(2 p^{2}\right)}$ and $z^{p} \in K_{(2 p)}$ by Lemma 7.1(iii). Let

$$
x_{1}=1+2 p \zeta, x_{2}=1+2 p\left(\zeta^{2}-\zeta^{3}+\zeta^{4}\right) .
$$

Then we get

$$
\begin{aligned}
h\left(\varphi^{*}\left(x_{1}\right)\right) & \left.\equiv h\left(1+2 p\left(\zeta+\zeta^{3}\right)\right)\right) \\
& \equiv\left[\begin{array}{cccc}
1-2 p & -2 p & -2 p & 0 \\
0 & 1-2 p & 0 & -2 p \\
2 p & 2 p & 1 & 0 \\
2 p & 4 p & 2 p & 1
\end{array}\right] \quad\left(\bmod 2 p^{2}\right),
\end{aligned}
$$

$$
h\left(\varphi^{*}\left(x_{2}\right)\right) \equiv h\left(1+2 p\left(\zeta+2 \zeta^{2}-\zeta^{3}\right)\right)
$$




$$
\equiv\left[\begin{array}{cccc}
1+2 p & 6 p & -2 p & 4 p \\
-4 p & 1-2 p & 4 p & -2 p \\
2 p & -2 p & 1-4 p & 4 p \\
-2 p & -4 p & -6 p & 1
\end{array}\right] \quad\left(\bmod 2 p^{2}\right)
$$

and

$$
\nu\left(h\left(\varphi^{*}\left(x_{1}\right)\right)\right) \equiv \nu\left(h\left(\varphi^{*}\left(x_{2}\right)\right)\right) \equiv 1-2 p \quad\left(\bmod 2 p^{2}\right) .
$$

Here we observe that $h\left(\varphi^{*}\left(x_{k}\right)\right)(k=1,2)$ belongs to $G_{2 p^{2}}$.

Lemma 7.2. Let $a, b, c, d \in \mathbb{Z}$.

(i) We have the formula

$$
\begin{gathered}
\Phi_{\left[\begin{array}{l}
a / p \\
b / p \\
c / p \\
d / p
\end{array}\right]}\left(Z_{0}\right)^{\left(\frac{K_{\left(2 p^{2}\right) / K}}{\left(x_{k}\right)}\right)} \quad(k=1,2) \\
e\left(\left(-a^{2}+2 a d-b^{2}-c^{2}-2 c d-2 d^{2}\right) / p\right) \Phi_{\left[\begin{array}{l}
a / p \\
b / p \\
c / p \\
d / p
\end{array}\right]}\left(Z_{0}\right) \quad \text { if } k=1, \\
e\left(\left(-a^{2}+4 a b-4 a c-b^{2}+4 b c-c^{2}+2 c d+2 d^{2}\right) / p\right) \Phi_{\left[\begin{array}{l}
a / p \\
b / p \\
c / p \\
d / p
\end{array}\right]}\left(Z_{0}\right) \text { if } k=2 .
\end{gathered}
$$

(ii) $\zeta_{2 p^{2}}^{\left(\frac{K_{\left(2 p^{2}\right) / K}}{\left(x_{k}\right)}\right)}=\zeta_{2 p^{2}}^{1-2 p}(k=1,2)$.

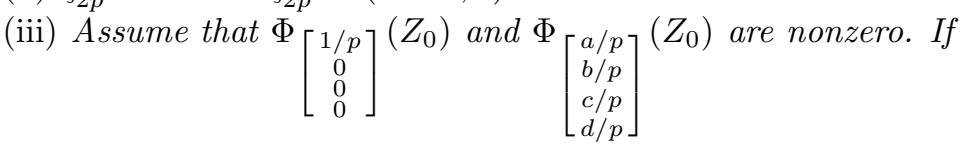

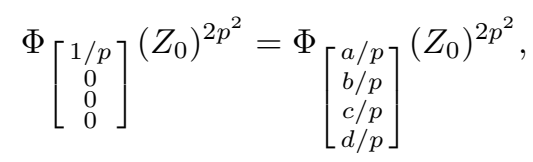

then $-2 a b+2 a c+a d-2 b c-2 c d-2 d^{2} \equiv 0(\bmod p)$.

Proof. (i) We derive that

$$
\begin{aligned}
& \Phi_{\left[\begin{array}{l}
a / p \\
b / p \\
c / p \\
d / p
\end{array}\right]}\left(Z_{0}\right)^{\left(\frac{K_{\left(2 p^{2}\right) / K}}{\left(x_{k}\right)}\right)} \quad(k=1,2) \\
= & \Phi_{\left[\begin{array}{l}
a / p \\
b / p \\
c / p \\
d / p
\end{array}\right]}(Z)^{h\left(\varphi^{*}\left(x_{k}\right)\right)}\left(Z_{0}\right) \quad \text { by Proposition } 6.2 \\
= & e\left(\left({ }^{t} \mathbf{r}(1-2 p) \mathbf{s}-{ }^{t} \mathbf{r}^{\prime} \mathbf{s}^{\prime}\right) / 2\right) \Phi_{\left[\begin{array}{l}
\mathbf{r}^{\prime} \\
\mathbf{s}^{\prime}
\end{array}\right]}\left(Z_{0}\right),
\end{aligned}
$$


where $\mathbf{r}=\left[\begin{array}{l}a / p \\ b / p\end{array}\right], \mathbf{s}=\left[\begin{array}{l}c / p \\ d / p\end{array}\right]$ and $\left[\begin{array}{l}\mathbf{r}^{\prime} \\ \mathbf{s}^{\prime}\end{array}\right]={ }^{t} h\left(\varphi^{*}\left(x_{k}\right)\right)\left[\begin{array}{l}\mathbf{r} \\ \mathbf{s}\end{array}\right]$,

by Remark 5.3(ii) and (11)

$=\left\{\begin{array}{l}e\left(\left(a^{2}+b^{2}-c^{2}-2 c d-2 d^{2}\right) / p\right) \Phi\left[\begin{array}{l}a / p \\ b / p \\ c / p \\ d / p\end{array}\right]+\left[\begin{array}{c}-2 a+2 c+2 d \\ -2 a-2 b+2 c+4 d \\ -2 a+2 d \\ -2 b\end{array}\right]\left(Z_{0}\right) \\ \text { if } k=1, \\ e\left(\left(a^{2}-4 a b+b^{2}-c^{2}+2 c d+2 d^{2}\right) / p\right) \Phi\left[\begin{array}{c}a / p \\ b / p \\ c / p \\ d / p\end{array}\right]+\left[\begin{array}{c}2 a-4 b+2 c-2 d \\ 6 a-2 b-2 c-4 d \\ -2 a+4 b-4 c-6 d \\ 4 a-2 b+4 c\end{array}\right] \\ \text { if } k=2,\end{array}\right.$

by (9), (10) and Lemma 5.1

$=\left\{\begin{array}{l}e\left(\left(-a^{2}+2 a d-b^{2}-c^{2}-2 c d-2 d^{2}\right) / p\right) \Phi_{\left[\begin{array}{l}a / p \\ b / p \\ c / p \\ d / p\end{array}\right]}\left(Z_{0}\right) \\ \text { if } k=1, \\ e\left(\left(-a^{2}+4 a b-4 a c-b^{2}+4 b c-c^{2}+2 c d+2 d^{2}\right) / p\right) \Phi_{\left[\begin{array}{l}a / p \\ b / p \\ c / p \\ d / p\end{array}\right]}\left(Z_{0}\right) \\ \text { if } k=2,\end{array}\right.$

by Proposition 3.1.

(ii) For $k=1,2$ we see that

$$
\begin{aligned}
\zeta_{2 p^{2}}^{\left(\frac{K\left(2 p^{2}\right)^{\prime K}}{\left(x_{k}\right)}\right)} & =\zeta_{2 p^{2}}^{h\left(\varphi^{*}\left(x_{k}\right)\right)} \quad \text { by Proposition } 6.2 \\
& =\zeta_{2 p^{2}}^{\left.\nu\left(\varphi^{*}\left(x_{k}\right)\right)\right)} \quad \text { by Proposition } 2.1 \\
& =\zeta_{2 p^{2}}^{1-2 p} \quad \text { by }(11) .
\end{aligned}
$$

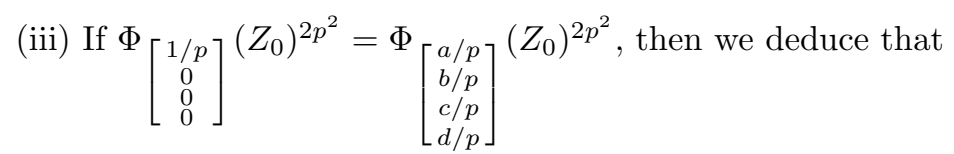

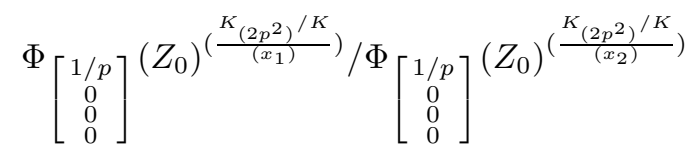

$$
\begin{aligned}
& =1 \text { by (i) } \\
& =\left(\xi \Phi_{\left[\begin{array}{l}
a / p \\
b / p \\
c / p \\
d / p
\end{array}\right]}\left(Z_{0}\right)\right)^{\left(\frac{K_{\left(2 p^{2}\right) / K}}{\left(x_{1}\right)}\right)} /\left(\xi \Phi_{\left[\begin{array}{l}
a / p \\
b / p \\
c / p \\
d / p
\end{array}\right]}\left(Z_{0}\right)\right)^{\left(\frac{K_{\left(2 p^{2}\right) / K}}{\left(x_{2}\right)}\right)}
\end{aligned}
$$


for some $2 p^{2}$ th root of unity $\xi$

$$
\begin{aligned}
& =\xi^{1-2 p} \Phi\left[\begin{array}{l}
a / p \\
b / p \\
c / p \\
d / p
\end{array}\right]\left(Z_{0}\right)^{\left(\frac{K_{\left(2 p^{2}\right) / K}}{\left(x_{1}\right)}\right)} / \xi^{1-2 p} \Phi_{\left[\begin{array}{c}
a / p \\
b / p \\
c / p \\
d / p
\end{array}\right]}\left(Z_{0}\right)^{\left(\frac{K_{\left(2 p^{2}\right) / K}}{\left(x_{2}\right)}\right)} \text { by (ii) } \\
& =e\left(\left(-4 a b+4 a c+2 a d-4 b c-4 c d-4 d^{2}\right) / p\right) \text { by (i). }
\end{aligned}
$$

This proves (iii).

Now we let

$$
\begin{aligned}
T=\left\{\sigma \in \operatorname{Gal}\left(K_{(2 p)} / K\right) \mid\right. & \left(z^{\sigma^{\prime}}\right)^{\left(\frac{K_{\left(2 p^{2}\right) / K}}{\left(x_{1}\right)}\right)}=\left(z^{\sigma^{\prime}}\right)^{\left(\frac{K_{\left(2 p^{2}\right) / K}}{\left(x_{2}\right)}\right)} \\
& \text { for some extension } \left.\sigma^{\prime} \text { of } \sigma \text { to } K_{\left(2 p^{2}\right)}\right\} .
\end{aligned}
$$

Since $K_{\left(2 p^{2}\right)} / K$ is an abelian extension, $T$ is obviously a subgroup of

$$
\operatorname{Gal}\left(K_{(2 p)} / K\right) \text {. }
$$

Furthermore, if $\sigma \in T$, then $\left(z^{\sigma^{\prime}}\right)^{\left(\frac{K\left(2 p^{2}\right) / K}{\left(x_{1}\right)}\right)}=\left(z^{\sigma^{\prime}}\right)^{\left(\frac{K\left(2 p^{2}\right) / K}{\left(x_{2}\right)}\right)}$ for all extensions $\sigma^{\prime}$ of $\sigma$ to $K_{\left(2 p^{2}\right)}$. Indeed, let $\sigma$ be an element of $T$ with some extension $\sigma^{\prime}$ satisfying $\left(z^{\sigma^{\prime}}\right)^{\left(\frac{K_{\left(2 p^{2}\right) / K}}{\left(x_{1}\right)}\right)}=\left(z^{\sigma^{\prime}}\right)^{\left(\frac{K_{\left(2 p^{2}\right) / K}}{\left(x_{2}\right)}\right)}$. Now that $z^{p} \in K_{(2 p)}$, if $\sigma^{\prime \prime}$ is another extension of $\sigma$ to $K_{\left(2 p^{2}\right)}$, then $\left(z^{p}\right)^{\sigma^{\prime \prime}}=\left(z^{p}\right)^{\sigma^{\prime}}$. So we get $z^{\sigma^{\prime \prime}}=\xi z^{\sigma^{\prime}}$ for some $p$ th root of unity $\xi$. And, since $\xi^{\left(\frac{K_{\left(2 p^{2}\right) / K}}{\left.x_{1}\right)}\right)}=\xi^{\left(\frac{K_{\left(2 p^{2}\right) / K}}{\left(x_{2}\right)}\right)}=\xi^{1-2 p}$ $(k=1,2)$ by Lemma 7.2(ii), we obtain that

$$
\left(z^{\sigma^{\prime \prime}}\right)^{\left(\frac{K\left(2 p^{2}\right) / K}{\left(x_{1}\right)}\right)}=\left(\xi z^{\sigma^{\prime}}\right)^{\left(\frac{K_{\left(2 p^{2}\right) / K}}{\left(x_{1}\right)}\right)}=\left(\xi z^{\sigma^{\prime}}\right)^{\left(\frac{K_{\left(2 p^{2}\right) / K}}{\left(x_{2}\right)}\right)}=\left(z^{\sigma^{\prime \prime}}\right)^{\left(\frac{K_{\left(2 p^{2}\right) / K}}{\left(x_{2}\right)}\right)} .
$$

Let $F$ be the subfield of $K_{(2 p)}$ fixed by $T$. Then by the Galois theory we see the isomorphism $\operatorname{Gal}\left(K_{(2 p)} / F\right) \simeq T$.

Theorem 7.3. $K\left(z^{2 p^{2}}\right)$ contains $F$.

Proof. By the Galois theory the assertion is equivalent to saying that

$$
\operatorname{Gal}\left(K_{(2 p)} / K\left(z^{2 p^{2}}\right)\right)
$$

is a subgroup of $T$. Let $\sigma \in \operatorname{Gal}\left(K_{(2 p)} / K\left(z^{2 p^{2}}\right)\right)$, that is, $\sigma$ is an element of $\operatorname{Gal}\left(K_{(2 p)} / K\right)$ which fixes $z^{2 p^{2}}$. If $\sigma^{\prime}$ is an extension of $\sigma$ to $K_{\left(2 p^{2}\right)}$, then we have $z^{\sigma^{\prime}}=\xi z$ for some $2 p^{2}$ th root of unity. Since both $\left(\frac{K_{\left(2 p^{2}\right)} / K}{\left(x_{1}\right)}\right)$ and $\left(\frac{K_{\left(2 p^{2}\right)} / K}{\left(x_{2}\right)}\right)$ send $\xi z$ to $\xi^{1-2 p} \zeta_{p}^{-1} z$ by Lemma 7.2(i) and (ii), $\sigma$ belongs to $T$. This proves the theorem.

Theorem 7.4. Let $x=a_{0}+a_{1} \zeta+a_{2} \zeta^{2}+a_{3} \zeta^{3}+a_{4} \zeta^{4}$ for integers $a_{0}, a_{1}, a_{2}, a_{3}, a_{4}$ such that $\mathrm{N}_{K / \mathbb{Q}}(x)$ is prime to $2 p$ and $h\left(\varphi^{*}(x)\right) \in G_{2 p^{2}}$. Assume that $z$ is 
nonzero. If $\left(\frac{K_{(2 p)} / K}{(x)}\right)$ fixes $z^{2 p^{2}}$, then we have $-2 a b+2 a c+a d-2 b c-2 c d-2 d^{2} \equiv$ $0(\bmod p)$, where

$$
\begin{aligned}
& a=a_{0}^{2}-a_{0} a_{1}-a_{0} a_{3}+a_{1} a_{2}+a_{1} a_{3}-a_{1} a_{4}-a_{2}^{2}+a_{2} a_{4}, \\
& b=-a_{0} a_{1}+a_{0} a_{2}-a_{0} a_{3}+a_{0} a_{4}+a_{1} a_{2}-a_{2}^{2}+a_{3}^{2}-a_{3} a_{4}, \\
& c=-a_{0} a_{1}-a_{0} a_{2}+a_{0} a_{3}+a_{0} a_{4}+a_{1}^{2}-a_{1} a_{3}+a_{2} a_{4}-a_{4}^{2}, \\
& d=a_{0} a_{2}-a_{0} a_{3}+a_{1} a_{3}-a_{1} a_{4}-a_{2}^{2}+a_{2} a_{3}-a_{3} a_{4}+a_{4}^{2} .
\end{aligned}
$$

Proof. One can readily verify that the first row of $h\left(\varphi^{*}(x)\right)$ is $\left[\begin{array}{llll}a & b & c & d\end{array}\right]$, namely

$$
\begin{aligned}
& x^{\varphi_{1}^{-1}} x^{\varphi_{2}^{-1}} \xi_{1} \\
= & \left(a_{0}+a_{1} \zeta+a_{2} \zeta^{2}+a_{3} \zeta^{3}+a_{4} \zeta^{4}\right)\left(a_{0}+a_{1} \zeta^{3}+a_{2} \zeta+a_{3} \zeta^{4}+a_{4} \zeta^{2}\right) \zeta^{2} \\
= & a \xi_{1}+b \xi_{2}+c \xi_{3}+d \xi_{4} .
\end{aligned}
$$

And we have

$$
\begin{aligned}
& \left(z^{2 p^{2}}\right)^{\left(\frac{K_{(2 p)} / K}{(x)}\right)}=\left(\Phi\left[\begin{array}{c}
1 / p \\
0 \\
0 \\
0
\end{array}\right](Z)^{2 p^{2}}\right)^{h\left(\varphi^{*}(x)\right)}\left(Z_{0}\right) \text { by Proposition } 6.2 \\
& =\Phi^{{ }^{t} h\left(\varphi^{*}(x)\right)}\left[\begin{array}{c}
1 / p \\
0 \\
0 \\
0
\end{array}\right](Z)^{2 p^{2}}\left(Z_{0}\right) \text { by Remark 5.3(ii) }
\end{aligned}
$$

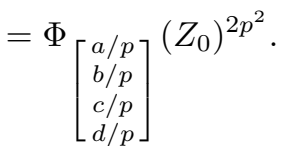

We then conclude the theorem by Lemma 7.2(iii).

Remark 7.5. Let $x$ be of the form $1+2 y$ for some $y \in \mathcal{O}_{K}$ such that $\mathrm{N}_{K / \mathbb{Q}}(x)$ is prime to $2 p$. Since

$$
h\left(\varphi^{*}(x)\right)=h\left((1+2 y)(1+2 y)^{\varphi_{2}^{-1}}\right) \equiv h(1) \equiv I_{2 g} \quad(\bmod 2),
$$

$h\left(\varphi^{*}(x)\right)$ belongs to $G_{2 p^{2}}$ automatically.

Example 7.6. We follow the notations and assumption in Theorem 7.4. Let $x=1+2\left(\zeta+\zeta^{2}\right)$, then $\mathrm{N}_{K / \mathbb{Q}}(x)=5$. So we assume $p \neq 5$. Since $a=-1$, $b=0, c=0, d=-2$, we get $-2 a b+2 a c+a d-2 b c-2 c d-2 d^{2} \equiv-6(\bmod p)$. Therefore, if $p \neq 3$, then $\left(\frac{K_{(2 p)} / K}{(x)}\right)$ does not fix $z^{2 p^{2}}$ by Theorem 7.4.

\section{Remarks on primitive generators}

In this section we shall introduce two useful methods of combining two generators of an abelian extension to get a primitive one. We begin with an example. 
Example 8.1. Following the same notations as in $\S 7$ we consider the field $K\left(z^{p}, \zeta_{25}\right)$. Note that $(2 z)^{p}$ is an algebraic integer which lies in $K_{(2 p)}$ by Lemma 7.1(ii) and (iii). The prime ideal $\left(1-\zeta_{5}\right) \mathcal{O}_{K}$ of $K$, which lies above the prime ideal $5 \mathbb{Z}$ of $\mathbb{Q}$, is totally ramified in the extension $K\left(\zeta_{25}\right) / K$ with ramification index $\left[K\left(\zeta_{25}\right): K\right]=\left[\mathbb{Q}\left(\zeta_{25}\right): \mathbb{Q}\right] /\left[\mathbb{Q}\left(\zeta_{5}\right): \mathbb{Q}\right]=5$. On the other hand, all prime ideals of $K$ which are ramified in $K_{(2 p)} / K$ must divide the ideal $2 p \mathcal{O}_{K}$. So we have $\left[K\left(z^{p}, \zeta_{25}\right): K\left(z^{p}\right)\right]=5$. Furthermore, a conjugate of $\zeta_{25}$ over $K\left(z^{p}\right)$ is of the form $\zeta_{25}^{1+5 k}(k=0,1,2,3,4)$. And we get

$$
\begin{aligned}
\operatorname{Tr}_{K\left(z^{p}, \zeta_{25}\right) / K\left(z^{p}\right)}\left(\zeta_{25}\right) & =\sum_{k=0}^{4} \zeta_{25}^{1+5 k}=\zeta_{25} \sum_{k=0}^{4} \zeta_{5}^{k}=0, \\
\mathrm{~N}_{K\left(z^{p}, \zeta_{25}\right) / K\left(z^{p}\right)}\left(3 \zeta_{25}+1\right) & =\prod_{k=0}^{4}\left(3 \zeta_{25}^{1+5 k}+1\right) \\
& =\left(-3 \zeta_{25}\right)^{5} \prod_{k=0}^{4}\left(-\zeta_{5}^{k}+\left(-3 \zeta_{25}\right)^{-1}\right) \\
& =243 \zeta_{5}+1 .
\end{aligned}
$$

We then obtain primitive generators of $K\left(z^{p}, \zeta_{25}\right)$ over $K$ by Theorems 8.2 and 8.4 as follows:

$$
K\left(z^{p}, \zeta_{25}\right)=K\left(z^{p}+\zeta_{25}\right)=K\left(\left(3(2 z)^{p}+1\right)\left(3 \zeta_{25}+1\right)^{-5}\left(243 \zeta_{5}+1\right)\right) .
$$

Theorem 8.2. Let $L$ be an abelian extension of a number field $K$. Suppose that $L=K(x, y)$ for some $x, y \in L$. Let $\ell=[L: K(x)]$, and $a$ and $b$ be any nonzero elements of $K$. Then we have

$$
L=K\left(a x+b\left(\ell y-\operatorname{Tr}_{L / K(x)}(y)\right)\right) .
$$

Proof. If we set $\varepsilon=a x+b\left(\ell y-\operatorname{Tr}_{L / K(x)}(y)\right)$, then we achieve

$$
\begin{aligned}
\operatorname{Tr}_{L / K(x)}(\varepsilon) & =\operatorname{Tr}_{L / K(x)}(a x)+\operatorname{Tr}_{L / K(x)}(b \ell y)+\operatorname{Tr}_{L / K(x)}\left(-b \operatorname{Tr}_{L / K(x)}(y)\right) \\
& =a x \operatorname{Tr}_{L / K(x)}(1)+b \ell \operatorname{Tr}_{L / K(x)}(y)-b \operatorname{Tr}_{L / K(x)}(y) \operatorname{Tr}_{L / K(x)}(1) \\
& =a x \ell .
\end{aligned}
$$

Observe that since $L / K$ is an abelian extension, any intermediate field of $L / K$ is an abelian extension of $K$ by Galois theory. This fact implies that $K(\varepsilon)$ contains $\varepsilon^{\sigma}$ for any $\sigma \in \operatorname{Gal}(L / K)$; whence $\operatorname{Tr}_{L / K(x)}(\varepsilon)$ belongs to $K(\varepsilon)$. Therefore we deduce that

$$
\begin{aligned}
K(\varepsilon) & =K(\varepsilon)\left(\operatorname{Tr}_{L / K(x)}(\varepsilon)\right)=K\left(\operatorname{Tr}_{L / K(x)}(\varepsilon)\right)(\varepsilon) \\
& =K(a x \ell)(\varepsilon)=K(x)(\varepsilon) \quad \text { by }(12) \\
& =K(x)\left(\varepsilon-a x+b \operatorname{Tr}_{L / K(x)}(y)\right)=K(x)(b \ell y)=K(x)(y)=L .
\end{aligned}
$$

This completes the proof. 
Lemma 8.3. Let $K$ be a number field and $x$ be an algebraic integer. Suppose that $K(x) / K$ is a Galois extension. If $a$ and $b$ are nonzero integers such that $2<|a / b|$, then we have

$$
K(x)=K\left((a x+b)^{n}\right) \quad \text { for a nonzero integer } n .
$$

Proof. We first note that $a x+b$ is nonzero. Otherwise, $x=-b / a$ is a nonzero rational number less than $1 / 2$, which contradicts the fact that $x$ is an algebraic integer. Suppose on the contrary $K(x) \supsetneq K\left((a x+b)^{n}\right)$. Then there exists a nontrivial element $\sigma$ of $\operatorname{Gal}\left(K(x) / K\left((a x+b)^{n}\right)\right)$. That is, $\left((a x+b)^{n}\right)^{\sigma}=$ $(a x+b)^{n}$ but $x^{\sigma} \neq x$, from which we see that $a x^{\sigma}+b=\xi(a x+b)$ for some $|n|$ th root of unity $\xi(\neq 1)$. Thus we have

$$
a\left(x^{\sigma}-\xi x\right)=b(\xi-1)(\neq 0) .
$$

Let $\ell=[K(x): \mathbb{Q}]$. Since $x^{\sigma}-\xi x$ is a nonzero algebraic integer, we derive that

$$
\left|\mathrm{N}_{K(x) / \mathbb{Q}}\left(a\left(x^{\sigma}-\xi x\right)\right)\right|=|a|^{\ell}\left|\mathrm{N}_{K(x) / \mathbb{Q}}\left(x^{\sigma}-\xi x\right)\right| \geq|a|^{\ell} .
$$

On the other hand, since any conjugate of $\xi-1$ over $\mathbb{Q}$ is of the form $\xi^{t}-1$ for some integer $t$ and $\left|\xi^{t}-1\right| \leq 2$, we claim that

$$
\left|\mathrm{N}_{K(x) / \mathbb{Q}}(b(\xi-1))\right|=|b|^{\ell}\left|\mathrm{N}_{K(x) / \mathbb{Q}}(\xi-1)\right| \leq|b|^{\ell} 2^{\ell} .
$$

Hence we get from (13) $|a|^{\ell} \leq|b|^{\ell} 2^{\ell}$, which contradicts the assumption $2<$ $|a / b|$. Therefore we conclude the lemma.

Theorem 8.4. Let $L$ be an abelian extension of a number field $K$. Suppose $L=K(x, y)$ for some algebraic integers $x$ and $y$. Let $\ell=[L: K(x)]$ and $a, b$, $c, d$ be nonzero integers such that $2<|a / b|$ and $2<|c / d|$. Then we have

$L=K\left((a x+b)^{n}(c y+d)^{-m \ell} \mathrm{N}_{L / K(x)}\left((c y+d)^{m}\right)\right)$ for nonzero integers $n$ and $m$.

Proof. If we let $\varepsilon=(a x+b)^{n}(c y+d)^{-m \ell} \mathrm{N}_{L / K(x)}\left((c y+d)^{m}\right)$, then we get that

(14) $\mathrm{N}_{L / K(x)}(\varepsilon)$

$$
\begin{aligned}
& =\mathrm{N}_{L / K(x)}\left((a x+b)^{n}\right) \mathrm{N}_{L / K(x)}\left((c y+d)^{-m \ell}\right) \mathrm{N}_{L / K(x)}\left(\mathrm{N}_{L / K(x)}\left((c y+d)^{m}\right)\right) \\
& =\mathrm{N}_{L / K(x)}\left((c y+d)^{-m \ell}\right)\left(\mathrm{N}_{L / K(x)}\left((c y+d)^{m}\right)\right)^{\ell} \\
& =(a x+b)^{n \ell} .
\end{aligned}
$$

Now that $L / K$ is an abelian extension, $K(\varepsilon)$ contains $\mathrm{N}_{L / K(x)}(\varepsilon)$. Thus we obtain that

$$
\begin{aligned}
K(\varepsilon) & =K(\varepsilon)\left(\mathrm{N}_{L / K(x)}(\varepsilon)\right)=K\left(\mathrm{~N}_{L / K(x)}(\varepsilon)\right)(\varepsilon) \\
& =K\left((a x+b)^{n \ell}\right)(\varepsilon) \quad \text { by }(14) \\
& =K(x)(\varepsilon) \quad \text { by Lemma } 8.3 \\
& =K(x)\left(\varepsilon /(a x+b)^{n} \mathrm{~N}_{L / K(x)}\left((c y+d)^{m}\right)\right. \\
& \left.=K(x)\left((c y+d)^{-m \ell}\right)\right) \\
& =K(x)(y)=L \quad \text { by Lemma 8.3. }
\end{aligned}
$$


This proves the theorem.

\section{References}

[1] E. de Shalit and E. Z. Goren, On special values of theta functions of genus two, Ann. Inst. Fourier (Grenoble) 47 (1997), no. 3, 775-799.

[2] J.-I. Igusa, On the graded ring of theta-constants. II, Amer. J. Math. 88 (1966), no. 1, 221-236.

[3] H. Y. Jung, J. K. Koo, and D. H. Shin, Ray class invariants over imaginary quadratic fields, Tohoku Math. J. 63 (2011), no. 3, 413-426.

[4] H. Klingen, Introductory Lectures on Siegel Modular Forms, Cambridge Studies in Advanced Mathematics, 20, Cambridge University Press, Cambridge, 1990.

[5] K. Komatsu, Construction of a normal basis by special values of Siegel modular functions, Proc. Amer. Math. Soc. 128 (2000), no. 2, 315-323.

[6] D. Kubert and S. Lang, Modular Units, Grundlehren der mathematischen Wissenschaften 244, Spinger-Verlag, 1981.

[7] S. Lang, Elliptic Functions, 2nd edition, Grad. Texts in Math. 112, Spinger-Verlag, New York, 1987.

[8] G. Shimura, On canonical models of arithmetic quotients of bounded symmetric domains, Ann. of Math. 91 (1970), 144-222.

[9] _ Introduction to the Arithmetic Theory of Automorphic Functions, Iwanami Shoten and Princeton University Press, Princeton, N. J., 1971.

[10] _ Theta functions with complex multiplication, Duke Math. J. 43 (1976), no. 4, $673-696$.

[11] On certain reciprocity-laws for theta functions and modular forms, Acta Math. 141 (1978), no. 1-2, 35-71.

[12] Abelian Varieties with Complex Multiplication and Modular Functions, Princeton Mathematical Series, 46., Princeton University Press, Princeton, N. J., 1998.

Department of Mathematics

HANKUK UNIVERSiTy OF Foreign Studies

YONGIN 449-791, KOREA

E-mail address: dhshin@hufs.ac.kr 